\title{
SUBSTELLAR OBJECTS IN NEARBY YOUNG CLUSTERS (SONYC). VIII. SUBSTELLAR POPULATION IN LUPUS 3*
}

\author{
Koraljka MuŽić ${ }^{1}$, Alexander Scholz ${ }^{2,3}$, Vincent C. Geers ${ }^{2}$, \\ Ray Jayawardhana ${ }^{4}$, AND Belén LóPez MarTí 5,6 \\ ${ }^{1}$ European Southern Observatory, Alonso de Córdova 3107, Casilla 19, Santiago 19001, Chile; kmuzic@eso.org \\ ${ }^{2}$ School of Cosmic Physics, Dublin Institute for Advanced Studies, 31 Fitzwilliam Place, Dublin 2, Ireland \\ ${ }^{3}$ School of Physics and Astronomy, St. Andrews University, North Haugh, St Andrews KY16 9SS, UK \\ ${ }^{4}$ Department of Astronomy and Astrophysics, University of Toronto, 50 St. George Street, Toronto, ON M5S 3H4, Canada \\ ${ }^{5}$ Centro de Astrobiología (INTA-CSIC), Departamento de Astrofísica, P.O. Box 78, E-28261 Villanueva de la Cañada, Madrid, Spain \\ ${ }^{6}$ Saint Louis University-Madrid Campus, Division of Science, Engineering and Nursing, Avenida del Valle 34, E-28003 Madrid, Spain \\ Received 2013 September 10; accepted 2014 March 3; published 2014 April 7
}

\begin{abstract}
SONYC-Substellar Objects in Nearby Young Clusters-is a survey program to investigate the frequency and properties of substellar objects in nearby star-forming regions. We present a new imaging and spectroscopic survey conducted in the young $(\sim 1 \mathrm{Myr})$, nearby ( $\sim 200 \mathrm{pc})$ star-forming region Lupus 3. Deep optical and near-infrared images were obtained with MOSAIC-II and NEWFIRM at the CTIO $4 \mathrm{~m}$ telescope, covering $\sim 1.4 \mathrm{deg}^{2}$ on the sky. The $i$-band completeness limit of $20.3 \mathrm{mag}$ is equivalent to $0.009-0.02 M_{\odot}$, for $A_{V} \leqslant 5$. Photometry and 11-12 yr baseline proper motions were used to select candidate low-mass members of Lupus 3 . We performed a spectroscopic follow-up of 123 candidates, using VIMOS at the Very Large Telescope, and we identify 7 probable members, among which 4 have spectral type later than M6.0 and $T_{\text {eff }} \leqslant 3000 \mathrm{~K}$, i.e., are probably substellar in nature. Two of the new probable members of Lupus 3 appear underluminous for their spectral class and exhibit emission line spectrum with strong $\mathrm{H}_{\alpha}$ or forbidden lines associated with active accretion. We derive a relation between the spectral type and effective temperature: $T_{\text {eff }}=(4120 \pm 175)-(172 \pm 26) \times$ SpT, where SpT refers to the $\mathrm{M}$ spectral subtype between 1 and 9. Combining our results with the previous works on Lupus 3, we show that the spectral type distribution is consistent with that in other star-forming regions, as well as the derived star-to-brown dwarf ratio of 2.0-3.3. We compile a census of all spectroscopically confirmed low-mass members with spectral type $\mathrm{M} 0$ or later.
\end{abstract}

Key words: brown dwarfs - stars: formation - stars: low-mass - stars: luminosity function, mass function

Online-only material: color figures

\section{INTRODUCTION}

SONYC-short for Substellar Objects in Nearby Young Clusters-is a comprehensive project aiming to provide a complete, unbiased census of substellar population down to a few Jupiter masses in young star-forming regions. Studies of the substellar mass regime at young ages are crucial to understanding the mass dependence in the formation and early evolution of stars and planets. Although the low-mass end of the initial mass function (IMF) has been the subject of intensive investigation over more than a decade, and by various groups, its origin is still a matter of debate (e.g., Bonnell et al. 2007; Bastian et al. 2010; Jeffries 2012). The relative importance of several proposed processes (dynamical interactions, fragmentation, accretion, photoevaporation) responsible for the formation of brown dwarfs (BDs) is not yet clear.

The SONYC survey is based on extremely deep optical and near-infrared wide-field imaging, combined with the Two Micron All Sky Survey (2MASS) and Spitzer photometry catalogs, which are correlated to create catalogs of substellar candidates and used to identify targets for extensive spectroscopic followup. In this work for the first time we also include a proper motion analysis, which greatly facilitates the candidate selection. Our observations are designed to reach mass limits well

\footnotetext{
* Based on observations collected at the European Southern Observatory under programs 087.C-0386 and 089.C-0432, and Cerro Tololo Inter-American Observatory's programs 2010A-0054 and 2011A-0144.
}

below $0.01 M_{\odot}$, and the main candidate selection method is based on the optical photometry. This way we ensure that a realistic picture of the substellar population in each studied cluster is obtained, avoiding the biases introduced by the mid-infrared selection (only objects with disks) or methane-imaging (only T-dwarfs). So far we have published results for three regions: NGC 1333 (Scholz et al. 2009, 2012a, 2012b), $\rho$ Ophiuchi (Geers et al. 2011; Mužić et al. 2012), and Chamaeleon-I (Mužić et al. 2011). We have identified and characterized more than 50 new substellar objects, among them a handful of objects with masses close to or below the Deuterium burning limit. Thanks to the SONYC survey and the efforts of other groups, the substellar IMF is now well characterized down to $\sim 5-10 M_{J}$, and we find that the ratio of the number of stars with respect to BDs lies between 2 and 6 . In NGC 1333 we find that, down to $\sim 5 M_{J}$, the free-floating objects with planetary masses are 20-50 times less numerous than stars, so that their total contribution to the mass budget is negligible.

In this paper we present the SONYC campaign in the Lupus 3 star-forming region. The Lupus dark cloud complex is located in the Scorpius-Centaurus OB association and consists of several loosely connected dark clouds showing different levels of star formation activity (see Comerón 2008 for a detailed overview). The main site of star formation within the complex is Lupus 3, which contains one of the richest associations of T-Tauri stars (Schwartz 1977; Krautter et al. 1997). The center of Lupus 3 is dominated by the two most massive members of the entire complex, the two Herbig Ae/Be stars known as HR 5999 and 
Table 1

Observing Log for Our Imaging and Spectroscopic Surveys

\begin{tabular}{|c|c|c|c|c|c|c|}
\hline Field No. & $\begin{array}{c}\alpha \\
(\mathrm{J} 2000)\end{array}$ & $\begin{array}{c}\delta \\
(\mathrm{J} 2000)\end{array}$ & Filter/Grism & UT Date & On-source Time & Instrument \\
\hline \multicolumn{7}{|c|}{ Imaging } \\
\hline 1 & $16: 10: 10$ & $-38: 49: 08$ & $i$ & 2010 Jun 12 & $8100 \mathrm{~s}$ & MOSAIC-II \\
\hline 2 & $16: 06: 53$ & $-38: 49: 37$ & $i$ & 2010 Jun 12 & $6900 \mathrm{~s}$ & MOSAIC-II \\
\hline 3 & $16: 10: 33$ & $-39: 22: 34$ & $i$ & 2010 Jun 13 & $7500 \mathrm{~s}$ & MOSAIC-II \\
\hline 4 & $16: 06: 56$ & $-39: 22: 16$ & $i$ & 2010 Jun 13 & $9000 \mathrm{~s}$ & MOSAIC-II \\
\hline 1 & $16: 11: 01$ & $-38: 38: 20$ & $J, K_{S}$ & 2010 Jun 15, 2010 Jun 16 & $450 \mathrm{~s}, 400 \mathrm{~s}$ & NEWFIRM \\
\hline 2 & $16: 08: 39$ & $-38: 38: 20$ & $J, K_{S}$ & 2010 Jun 15, 2010 Jun 16 & $450 \mathrm{~s}, 400 \mathrm{~s}$ & NEWFIRM \\
\hline 3 & $16: 06: 18$ & $-38: 38: 20$ & $J, K_{S}$ & 2010 Jun 15, 2011 May 5 & $450 \mathrm{~s}, 420 \mathrm{~s}$ & NEWFIRM \\
\hline 4 & $16: 11: 01$ & $-39: 05: 56$ & $J, K_{S}$ & 2010 Jun 15, 2011 May 5 & $450 \mathrm{~s}, 400 \mathrm{~s}$ & NEWFIRM \\
\hline 5 & $16: 08: 39$ & $-39: 05: 56$ & $J, K_{S}$ & 2010 Jun 15, 2011 May 5 & $480 \mathrm{~s}, 460 \mathrm{~s}$ & NEWFIRM \\
\hline 6 & $16: 06: 18$ & $-39: 05: 56$ & $J, K_{S}$ & 2010 Jun 15, 2011 May 5 & $450 \mathrm{~s}, 420 \mathrm{~s}$ & NEWFIRM \\
\hline 7 & $16: 11: 01$ & $-39: 33: 32$ & $J, K_{S}$ & 2010 Jun 15,2011 May 5 & $450 \mathrm{~s}, 440 \mathrm{~s}$ & NEWFIRM \\
\hline 8 & $16: 08: 39$ & $-39: 33: 32$ & $J, K_{S}$ & 2010 Jun 15, 2011 May 5 & $450 \mathrm{~s}, 440 \mathrm{~s}$ & NEWFIRM \\
\hline$\underline{9}$ & $16: 06: 39$ & $-39: 33: 32$ & $J, K_{S}$ & 2010 Jun 15, 2011 May 5 & $450 \mathrm{~s}, 460 \mathrm{~s}$ & NEWFIRM \\
\hline \multicolumn{7}{|c|}{ Spectroscopy } \\
\hline 1 & $16: 11: 24$ & $-39: 01: 12$ & LR_red & 2011 Jun 26 & $3500 \mathrm{~s}$ & VIMOS \\
\hline 2 & $16: 11: 18$ & $-39: 01: 12$ & LR_red & 2012 Jun 12 & $3500 \mathrm{~s}$ & VIMOS \\
\hline 3 & $16: 11: 00$ & $-39: 04: 48$ & LR_red & 2012 Jun 16 & $3500 \mathrm{~s}$ & VIMOS \\
\hline 4 & $16: 09: 30$ & $-39: 01: 12$ & LR_red & 2012 Jun 27 & $3500 \mathrm{~s}$ & VIMOS \\
\hline 5 & $16: 08: 33$ & $-39: 05: 24$ & LR_red & 2012 Jul 15 & $3500 \mathrm{~s}$ & VIMOS \\
\hline 6 & $16: 07: 19$ & $-39: 07: 30$ & LR_red & 2012 Jun 17 & $3500 \mathrm{~s}$ & VIMOS \\
\hline
\end{tabular}

HR 6000. More than half of the known Lupus 3 members are found in the $0.3 \times 0.3 \mathrm{pc}^{2}$ area surrounding the pair (Nakajima et al. 2000; Comerón 2008).

The low-mass (sub-)stellar content of Lupus 3 was extensively investigated using the data from the Spitzer Space Telescope. Merín et al. (2008) compiled the most complete census of stars and BDs in Lupus 3 at the time, using the data from their survey together with the results of previous surveys by Nakajima et al. (2000), Comerón et al. (2003), Comerón (2008), López Martí et al. (2005), Allers et al. (2006), Allen et al. (2007), Chapman et al. (2007), Tachihara et al. (2007), Merín et al. (2007), Strauss et al. (1992), and Gondoin (2006). Spectroscopic followup in the optical (FLAMES/Very Large Telescope (VLT)) by Mortier et al. (2011) confirmed the effectiveness of MIR-excess selection from Merín et al. (2008), with about $80 \%$ of the 46 observed sources confirmed as members of Lupus 3. Two widearea photometric surveys in Lupus were conducted using the Wide-Field Imager (WFI) at the La Silla $2.2 \mathrm{~m}$ telescope. López Martí et al. (2005) identified 22 new low-mass member candidates in an area of $1.6 \mathrm{deg}^{2}$ in Lupus 3, with about half of the candidates confirmed spectroscopically in surveys by Allen et al. (2007) and Mortier et al. (2011). Comerón et al. (2009) surveyed an area of more than $6 \mathrm{deg}^{2}$ in the Lupus 1, 3, and 4 clouds and identified $\sim 70$ new candidate members of Lupus 3. About $50 \%$ of the photometric sample was revealed to belong to a background population of giant stars in a follow-up spectroscopic study by Comerón et al. (2013). The deepest survey so far in Lupus 3 (Comerón 2011) did not report any BDs, despite the sensitivity to objects with sub-Jupiter masses. However, the surveyed area of $\sim 7^{\prime} \times 7^{\prime}$ was very small compared with the total extent of the cluster and thus contained only a minor fraction of the total population. The substellar population in Lupus 3 remains therefore poorly constrained.

The distance to the Lupus star-forming region is still a matter of debate, with the distance determinations from different studies ranging from $140 \mathrm{pc}$ to $240 \mathrm{pc}$ (Comerón 2008). A widely used value of $140 \pm 20 \mathrm{pc}$ was derived by Hughes et al. (1993) using photometry and spectroscopy of F-G field stars in Lupus. Using an improved version of the same method, Lombardi et al. (2008) obtained a distance of $155 \pm 8$ pc but also suggest that the apparent thickness of Lupus might be the result of different Lupus sub-clouds being at different distances. Distances obtained from the moving-cluster method (Makarov 2007) suggest a thickness of $\sim 80 \mathrm{pc}$ and place Lupus 3 at a distance of about $170 \mathrm{pc}$, or $25 \mathrm{pc}$ farther than the center of the greater association. This spatial arrangement is in general agreement with the distances derived from Hipparcos parallaxes of individual stars located in different sub-clouds (Wichmann et al. 1998; Bertout et al. 1999). As pointed out by Comerón (2008), while a distance of 150 pc seems adequate for most of the clouds of the complex, a value of $200 \mathrm{pc}$ is likely to be more appropriate for Lupus 3 . We therefore adopt a distance of $200 \mathrm{pc}$ throughout the analysis presented in this paper. Adopting the distance of $200 \mathrm{pc}$, and evolutionary tracks of Baraffe et al. (1998) and Comerón et al. (2003) derive an age of 1-1.5 Myr for most of their observed members.

In this work we present new observations of the Lupus 3 cloud using the MOSAIC-II optical imager and NEWFIRM nearinfrared imager at the CTIO $4 \mathrm{~m}$ telescope. Observations and data reduction are explained in Section 2. Photometry, proper motions, and criteria for candidate selection are presented in Section 3 and the spectral analysis in Section 4. The results are discussed in Section 5. Finally, we summarize the main conclusions in Section 6.

\section{OBSERVATIONS AND DATA REDUCTION}

\subsection{Optical Imaging}

The imaging in the $i$ band was conducted using the MOSAICII CCD imager at the CTIO $4 \mathrm{~m}$ telescope in Chile. MOSAIC-II is a wide-field imager with eight CCDs arranged in a $4 \times 2$ array. It provides a field of view (FOV) of 36.8 on a side, with a pixel scale of $0^{\prime} .27$ pixel $^{-1}$. We observed four pointings arranged around the two bright Herbig Ae/Be stars. The details of the 

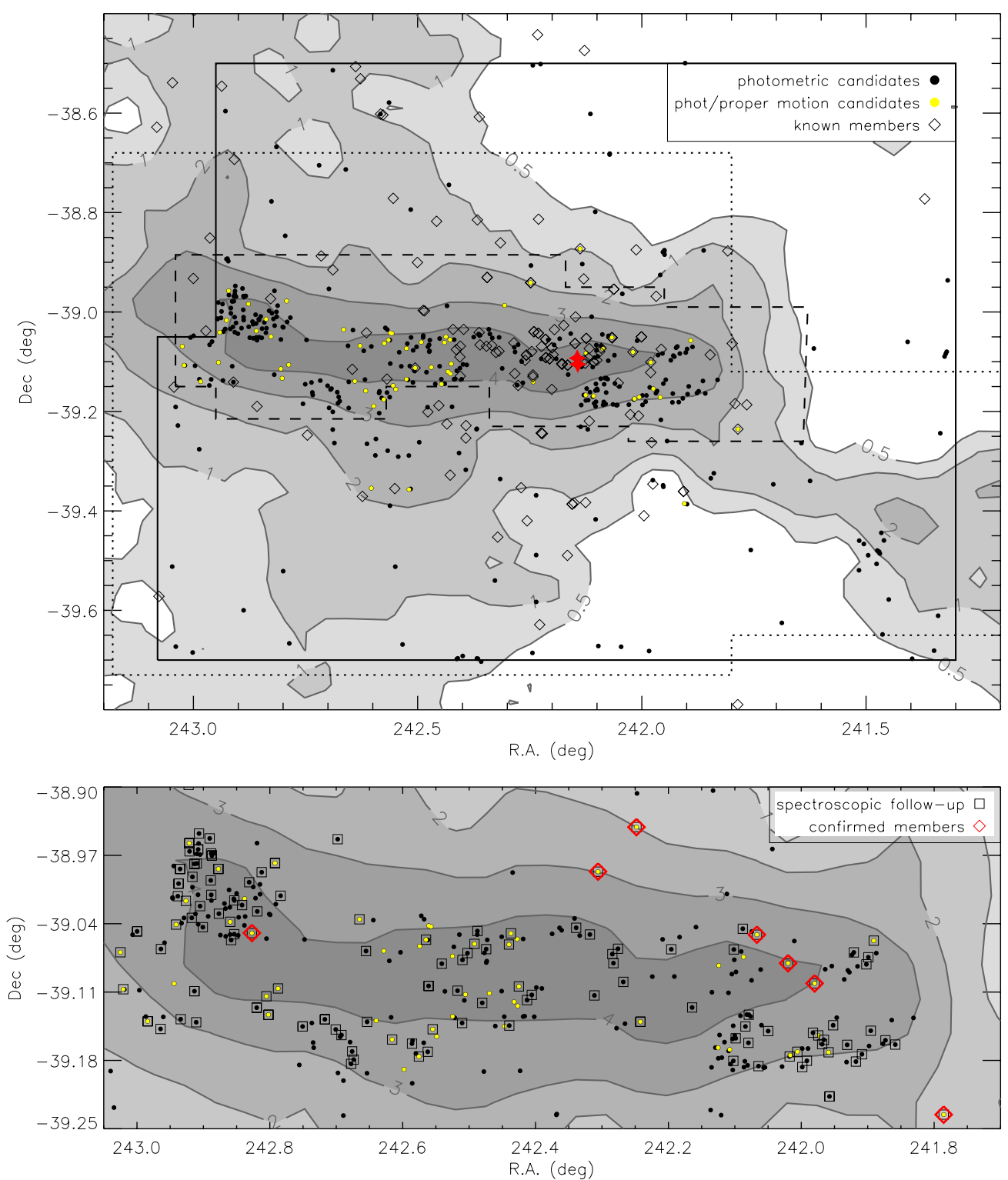

Figure 1. Upper panel: spatial distribution of the candidate sources in Lupus 3. Photometric candidates are shown as black dots, and those also selected by proper motions are shown in yellow. Diamonds mark previously known members, with the two red stars marking the brightest pair, HR5999/6000. The solid line shows the field of view of our optical and near-infrared survey, and the dotted line shows the extent of the WFI field used as the first epoch for the proper motions. The dashed line indicates the region within which the VIMOS spectroscopic fields have been arranged. The shaded contours indicate $A_{V}$ from the extinction map by Cambrésy (1999). Lower panel: zoom into the region covered by spectroscopy. All the candidates observed in our follow-up are marked with squares, and the confirmed objects are represented by red diamonds.

(A color version of this figure is available in the online journal.)

observing run are summarized in Table 1, and the observed field is shown in Figure 1.

We reduced the MOSAIC-II images using tools in the mscred package within IRAF. Mscred contains the standard reduction tasks for CCD images but adapted for the use on multi-chip mosaics. Series of dark frames and dome flats were combined to build an average zero and flat-field image. The zero and flatfield correction of the science mosaics was then carried out using the task "ccdproc." This also includes the subtraction of the bias level, derived from unexposed areas of the chip. For each of the four fields, the total integration time was split in a series of 24 exposures. To coadd these exposures, we first derived matching coordinate systems for all of them, using a list of USNO-A2 stars (obtained from Vizier) as reference. The frames were then matched and stacked using the tasks "mscimage" and "mscstack." Apart from a small region close to the center of the mosaic, which was excluded, this matching algorithm produces very good results. Note that the depth of the mosaics is not constant because of the small gaps between the detectors.

Photometry on the stacked images was carried out using "daophot" tools within IRAF. After generating a source catalog with "daofind." the magnitudes were measured within a fixed aperture radius of 10 pixels using "phot." The sky background was estimated from an annulus with an inner and outer radius of 10 and 15 pixels, respectively. To calibrate the $i$-band photometry, we compared our instrumental magnitudes with the magnitudes of sources with $14.5<i<17.0$ in Lupus 3 from the Deep Near Infrared Survey (DENIS) catalog (Epchtein et al. 1994), which used a Gunn $i$ filter centered at $0.8 \mu \mathrm{m}$. For each field we calculate a zero-point and apply it to all sources to create a final $i$-band catalog. The color dependence of the calibration was investigated by calculating a coefficient $a$ in the 
color term $a \times(i-J){ }^{7}$ which typically gives $a \sim 0.002$. Its effect on photometry would be much smaller than the involved photometric uncertainties, and therefore it was not taken into account. Photometric uncertainties combine the errors returned by "daophot," with the errors of the photometric calibration. Typical photometric uncertainties are $0.05 \mathrm{mag}$ at $i=19$ and 0.1 at $i=21$. The completeness limit of the deep exposures is $i \sim 20.3$, with the magnitude range of $\sim 15.3-22$.

For the proper motion calculation (see Section 3.2), we also used the $I_{C}$-band data obtained with the WFI at the MPIESO $2.2 \mathrm{~m}$ telescope at La Silla Observatory (Baade et al. 1999), which were previously published in López Martí et al. (2005). We therefore refer the reader to that paper for a detailed description of the data reduction procedures. The field observed with WFI is outlined with a dotted line in Figure 1.

\subsection{Near-infrared Imaging}

The near-infrared observations were designed to provide $J$ - and $K_{S}$-band photometry in the area slightly larger than the one covered with MOSAIC-II. We used NEWFIRM at the CTIO $4 \mathrm{~m}$ telescope, providing an FOV of $28^{\prime} \times 28^{\prime}$ and a pixel scale of 0'. 4 . The NEWFIRM detector is a mosaic of four $2048 \times 2048$ Orion InSb arrays, organized in a $2 \times 2$ grid. See Table 1 for the details of the NEWFIRM observing runs. Data reduction was performed using the NEWFIRM pipeline. The data were dark- and sky-subtracted and were corrected for bad pixels and flat-field effects. The astrometry was calibrated with respect to the 2MASS (Skrutskie et al. 2006) coordinate system, and each detector quadrant was re-projected onto an undistorted celestial tangent plane. The four quadrants were then combined into a single image, followed by the final stacking into mosaics. Source extraction was performed using SExtractor, requiring at least 5 pixels with flux above the $3 \sigma$ detection limit, followed by the rejection of sources at the edges of the detector and overly elongated objects $(a / b>2.0)$. Photometric zero-points for each NEWFIRM field were calculated by matching the sources in our catalog with the sources found in 2MASS. The completeness limits of the $J$ - and $K_{S}$-band catalogs are at $J=$ $18.3 \mathrm{mag}$ and $K_{S}=17.6$. Typical photometric uncertainties are $\leqslant 0.05 \mathrm{mag}$ at $J \leqslant 18$ and $K_{S} \leqslant 17$ and $0.1 \mathrm{mag}$ at $J=19$ and $K_{S}=18$.

\subsection{Multi-object Spectroscopy}

We obtained optical spectra using VIMOS/VLT in the MultiObject Spectroscopy (MOS) mode in programs 087.C-0386 and 089.C-0432. One field was observed in P87 and five in P89. ${ }^{8}$ VIMOS is a wide-field imager with four CCDs arranged in a $2 \times 2$ array. Each detector covers an FOV of $7^{\prime} \times 8^{\prime}$ with a pixel resolution of $0{ }^{\prime} .205$. The four quadrants are separated by gaps of approximately $2^{\prime}$. The spectra were obtained using the low-resolution red grism (LR_red). We covered the wavelength regime between $5500 \AA$ and $9500 \AA$, with the dispersion of $7.1 \AA$ pixel $^{-1}$ and slit width of $1^{\prime \prime}$, resulting in a spectral resolution of $R \sim 210$. The total on-source time for each field was $\sim 3500 \mathrm{~s}$. The dashed line in Figure 1 outlines the area where the VIMOS fields have been arranged.

Data reduction was performed using the VIMOS pipeline provided by ESO. Data reduction steps include bias subtraction, flat-field and bad-pixel correction, and wavelength and flux

\footnotetext{
$7 J$-band photometry from NEWFIRM data (Section 2.2).

8 ESO's period P87 denotes a period 2011 April-September and P89 denotes 2012 April-September.
}

calibration, as well as the final extraction of the spectra. The extraction is done by applying the optimal extraction from Horne (1986), which averages the signal optimally weighted by a function of the signal noise. In total we obtained 124 spectra. For the description of the selection criteria for spectroscopy, see Section 3.

\section{CANDIDATE SELECTION}

We start this section with a summary of the candidate selection procedure. All individual steps are in detail explained in the following subsections.

1. Our parent sample comprises the $\sim 500,000$ sources in the $i J$ photometric catalog created from the MOSAIC and NEWFIRM observations.

2. $(i, i-J)$ photometric selection results in 409 candidates (see Section 3.1).

3. We further narrowed the sample of $(i, i-J)$ photometric candidates from 409 to 59, by applying the proper motion selection criterion (see Section 3.2). In the following sections, we refer to this sample as " $I J$-pm".

4. To complement our main optical-NIR selection, we selected mid-infrared excess sources using Spitzer IRAC colors (75 candidates). This list contains 19 proper motion candidates (see Section 3.3). We refer to this sample as "IRAC-pm".

Section 4 describes the selection of targets for spectroscopic follow-up from the above mentioned candidate lists.

\subsection{Optical and Near-infrared Photometric Selection}

Figure 2 shows the $(i, i-J)$ color-magnitude diagram (CMD) of the sources observed in Lupus 3. Very low mass (VLM) objects are expected to occupy a distinct region on the red side of the broad cumulation of the background main-sequence stars. As in all the previous SONYC works, we construct the selection box in the CMD such that it represents a continuation of the sequence of the known members. We note that the majority of the known members are brighter than $i=15.5$ and are thus located outside the range of the CMD shown in Figure 2 (the saturation level of our optical data set is around $i=15.3 \mathrm{mag}$ ). Green stars show a subset of known spectroscopic members from Mortier et al. (2011), Comerón et al. (2013) fainter than $i=14.0$, with $i$ and $J$ photometry from DENIS and 2MASS, respectively. For clarity, below $i=15.3$ mag we show only the three members that overlap with the sources in our selection box (green stars).

For comparison we show DUSTY00 (Chabrier et al. 2000) and BT-Settl (Allard et al. 2011) isochrones for $1 \mathrm{Myr}$, adjusted to the distance of Lupus 3. The isochrones ${ }^{9}$ are shown in the same photometric filters (DENIS and 2MASS) as our data. According to the BT-Settl isochrone, the majority of the lowmass members are expected to be within our selection box, while looking at DUSTY we might be missing some objects with masses above $0.02 M_{\odot}$. However, it has to be taken into account that the isochrones have no extinction applied to them, while the potential members of Lupus are expected to have a certain degree of extinction, i.e., we expect them to be located to the right of the isochrones.

In total, 409 sources were found within the selection box and served as the initial list of candidates for spectroscopic

\footnotetext{
9 Available at http://phoenix.ens-lyon.fr/Grids/.
} 


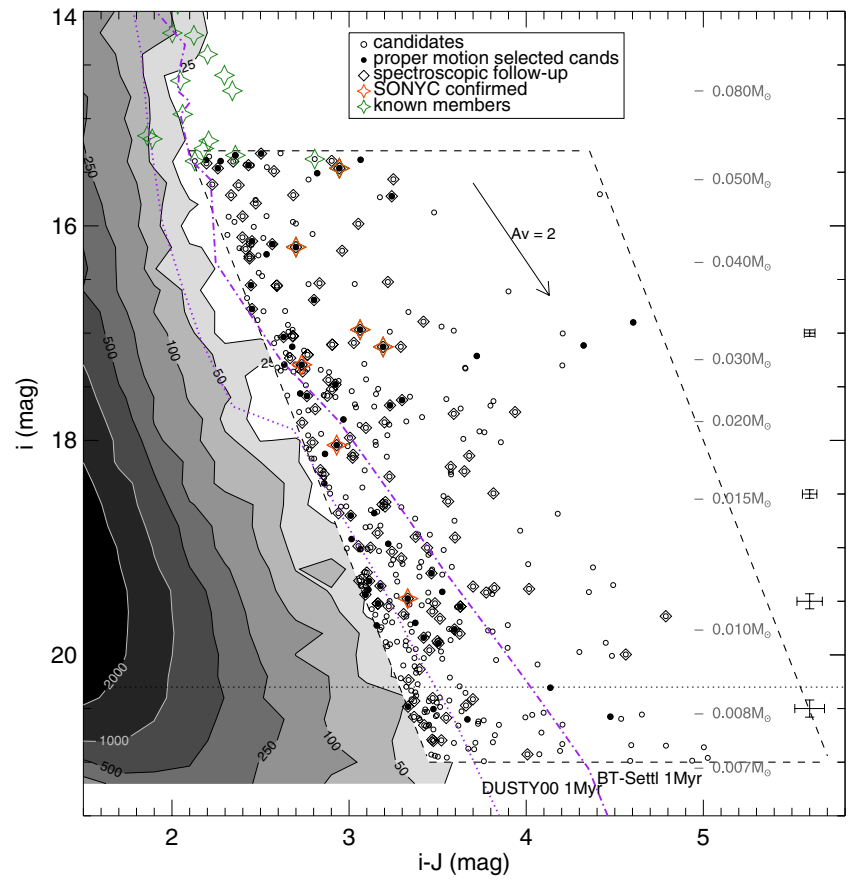

Figure 2. $(i, i-J)$ color-magnitude diagram. Open circles represent all the photometrically selected candidates located inside the selection box (dashed lines), with those additionally selected on the basis of their proper motions shown as filled circles. Diamonds show SONYC spectroscopy follow-up targets, with spectroscopically confirmed VLMOs highlighted as red (identified in this work) and green (previously known members) stars. Atmosphere model isochrones for age 1 Myr are overplotted for DUSTY00 (Chabrier et al. 2000) and BT-Settl (Allard et al. 2011). For clarity, the sources outside the candidate selection box are represented with contours, where the number on each contour represents the number of sources within a $0.2 \mathrm{mag} \times 0.2 \mathrm{mag}$ bin. On the right-hand side of the figure we show $1 \sigma$ uncertainties of the photometry. The dotted line shows the completeness limit of our survey.

(A color version of this figure is available in the online journal.)

follow-up with VIMOS. Further selection was performed based on the proper motion measurements, as described in the following section.

\subsection{Proper Motions}

Proper motion measurements are based on the WFI data set obtained between 1999 May 28 and June 3 and our NEWFIRM data from 2010 June and 2011 May. Whenever possible, we use the data from 2011 to ensure the longest time baseline. In the following we describe in detail all the steps performed.

1. The overlapping fields were first divided into sub-regions with the maximum size of $3^{\prime} \times 3^{\prime}$ and with an overlap of 0.5 between the adjacent fields. We find that an area of this size typically contains a high number of sources that can be used for astrometric transformations, while being reasonably small to minimize the effects of field distortions.

2. The absolute coordinate calibration shows systematic offsets of up to $12^{\prime \prime}$ between epochs in some regions, which poses a problem with cross-referencing individual sources, given the on-sky source density in Lupus 3. It is therefore crucial to apply an initial offset to the coordinates in one of the epochs to ensure the correct source registration in the two epochs. To determine the offset for each sub-region created in the first step, we create two images of the same size as the NEWFIRM and WFI sub-region of interest. The images have a value of 1 at the positions equivalent to the centroid pixels of bright stars and a value of zero elsewhere. These images are then cross-correlated to obtain an average shift applied to a particular region.

3. In this step we cross-match the catalogs on the basis of right ascension and declination. This step is used only to identify individual sources in the two epochs but not to calculate proper motions. The maximum matching radius of $2^{\prime \prime}$ was

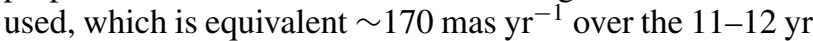
baseline.

4. We apply a cutoff in magnitude for the matching sources in order to discard saturated and faint ones (we keep all the sources with $J \approx 15.5-17.5$ ). The remaining sources are used in the next step to calculate a transformation of WFI positions to NEWFIRM coordinates. The number of sources used for the transformation in each sub-region varies between 80 and 400, with an average of 240 .

5. In the final step, we calculate a polynomial that describes the transformation of the WFI detector $(x, y)$ positions into the NEWFIRM coordinate frame for each sub-region, using the IDL procedure POLYWARP. In order to avoid the uncertainties in the absolute coordinate calibration, this step was performed in detector coordinates relative from one data set to the other. We tested a second- and a thirddegree polynomial and find that the two transformations give very similar final results, which is expected given the large number of sources available to calculate the transformation. Any polynomial with a degree $\geqslant 2$ corrects for shift and rotation between the fields and also largely compensates for the distortions of the two detectors (e.g., Schödel et al. 2009). After applying the transformation, a proper motion was calculated for each source. Since the vast majority of stars used to calculate the transformation between the epochs are not Lupus members, we expect a circularly symmetric distribution around $(0,0)$ in the proper motion space. We note that some of the members might have been included in the transformation, but their number compared with the total number of sources in our survey area is negligible and thus does not influence the results. Finally, proper motions for the sources toward Lupus 3 are shown as black dots in Figure 3.

In order to assess the uncertainties of the proper motion measurements, we use the fact that the chips were divided into overlapping sub-regions in step (1) described above. This allows us to derive the uncertainties by comparing proper motions of common sources that were obtained using different sets of reference stars. For each NEWFIRM field, we adopt a common uncertainty calculated as a standard deviation of all the differences obtained from the overlapping regions in each field. For the sources with more than one measurement, the average is adopted as the final proper motion value.

López Martí et al. (2011) performed a kinematic study of the bright objects in the Lupus regions and found the average proper motion of the Lupus 3 members to be $v_{\alpha}=$

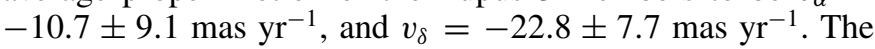
uncertainties quoted here represent the standard deviation of the measurements for Lupus 3 given in Table 2 of López Martí et al. (2011). We select all the sources within an ellipse centered at $(-10.7,-22.8)$ mas $\mathrm{yr}^{-1}$ and with the semi-major axes equal to two times the above quoted uncertainties. This list was crossmatched with the photometric candidate catalog described in the previous section, resulting finally in the list of 59 highpriority candidate members ("IJ-pm" sample; shown as filled black circles in Figure 2). The proper motions of the members 

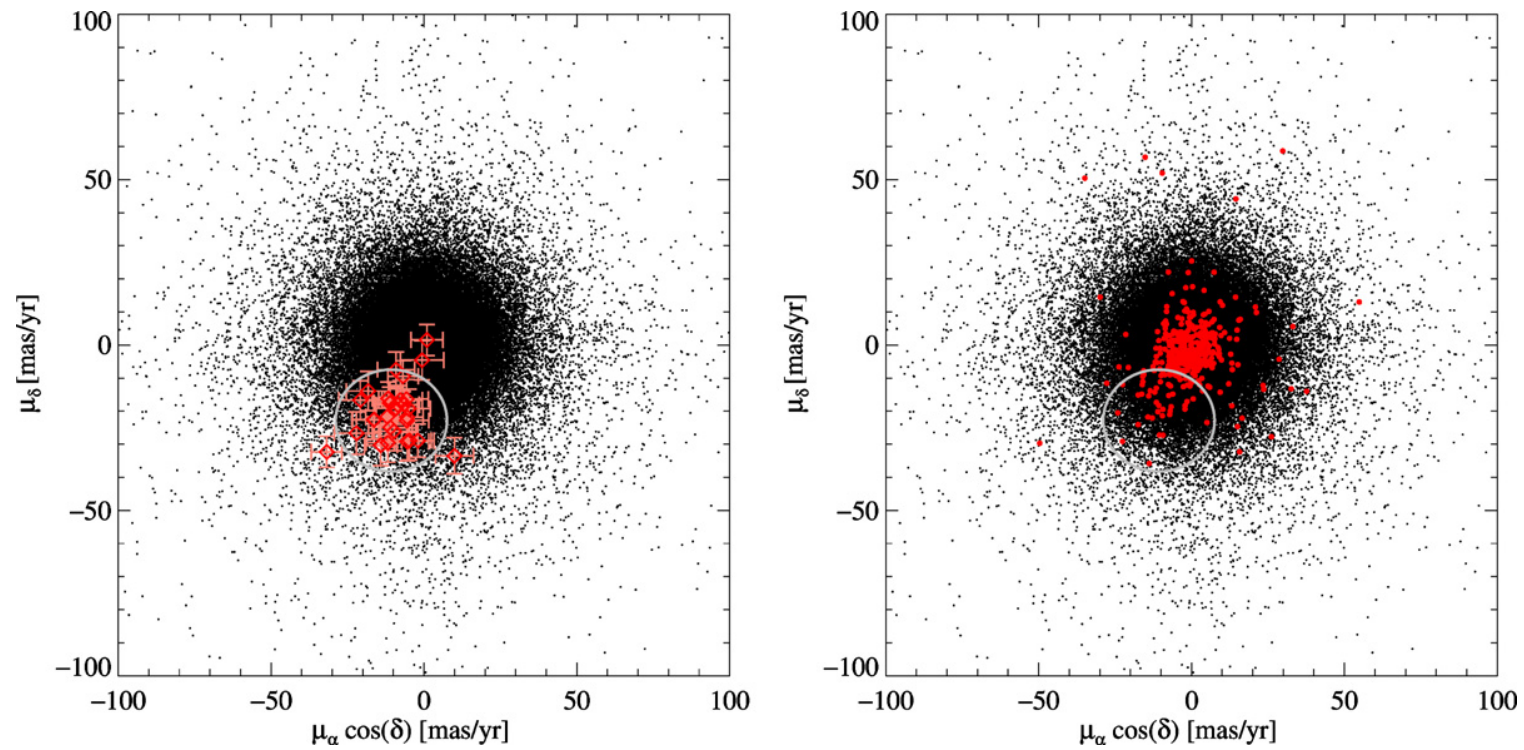

Figure 3. Proper motions of objects in the Lupus 3 field, based on 11-12 yr baseline between the WFI and NEWFIRM data (black dots). Left: the red symbols show the measured proper motions for the sources identified as members in López Martí et al. (2011). The ellipse depicts the $2 \sigma$ criterion used for the proper motion candidate selection (see Section 3.2). Right: red dots mark the proper motions of our 409 candidates selected from optical and NIR photometry. There are 59 candidates that pass both photometric and proper motion selection criteria.

(A color version of this figure is available in the online journal.)
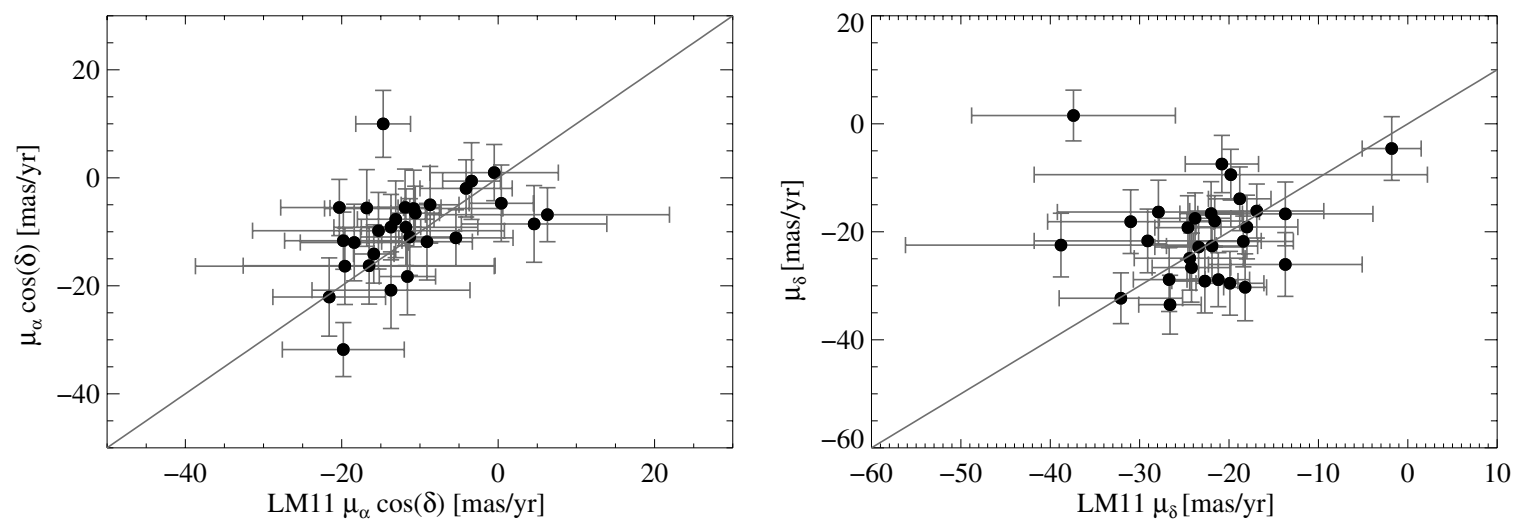

Figure 4. Comparison of the proper motions of the Lupus 3 members from López Martí et al. (2011) and this work. A line $x=y$ is shown as a solid line.

in common with López Martí et al. (2011) are shown as red symbols in the left panel of Figure 3, on top of the circular proper motion distribution of all other sources in our field (black dots). The right panel shows the proper motions of the photometric candidates identified in this survey (red points).

Figure 4 shows a comparison between the proper motions of the Lupus 3 members from López Martí et al. (2011) and the proper motions of these same sources obtained in this work. There are in total 40 matching objects. While virtually all of these sources are saturated in WFI images, the brightest ones show severe saturation effects such as strong CCD leaking, which can seriously affect the astrometry. We therefore discard the extremely saturated sources with $I_{C}>12.2$. The agreement between the two sets of proper motions is satisfactory, with all proper motions agreeing within $3 \sigma$.

\subsection{Mid-infrared Selection}

We retrieved the Spitzer IRAC and MIPS photometry of Lupus 3 from the Legacy Program data archive available at the Spitzer Science Center, using the "High reliability catalog" created by the "Cores to Disk" (c2d) Legacy team, made available at the SSC Web site. ${ }^{10}$

The Spitzer catalog was cross-correlated with our $i$-band catalog, requiring the separation of 2 " or better, and uncertainties $\leqslant 0.2 \mathrm{mag}$ in the first four IRAC bands. To select the MIR-excess sources, we require $I 3-I 4 \geqslant 0.4$ and $I 1-I 2>0$. This selection contains 75 sources, shown in Figure 5 as dots to the right of the $I 3-I 4 \geqslant 0.4$ line. Squares mark the 11 sources selected for follow-up spectroscopy. Of the 75 Spitzer-IRAC candidates, there are 19 sources that satisfy the proper motion criterion applied to the $i J$ candidate list and were included in the high-priority candidate list supplied to the VIMOS allocation software (we name this list the "IRAC-pm" sample). The Spitzer-IRAC list and the iJ photometric candidate list have seven objects in common.

\section{SPECTRAL ANALYSIS}

Slits were allocated to 123 out of 409 objects, selected from the optical CMD, using the VIMOS Mask Preparation Software

\footnotetext{
${ }^{10}$ http://data.spitzer.caltech.edu/popular/c2d/20071101_enhanced_v1/ lupus/catalogs/
} 


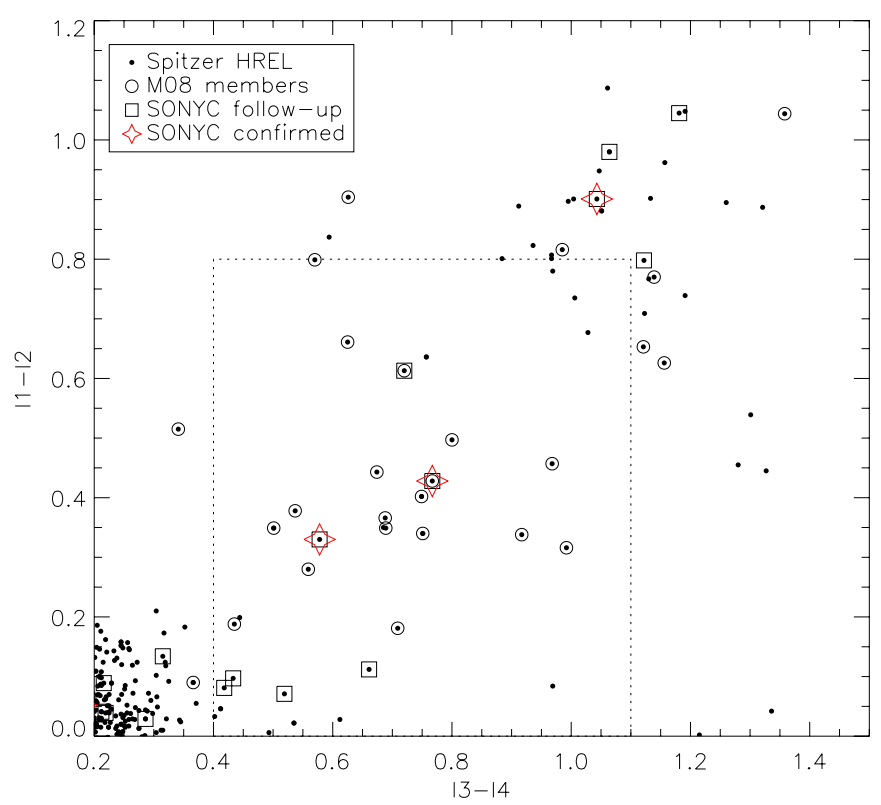

Figure 5. [3.6]-[4.5], [5.8]-[8.0] diagram constructed from Spitzer IRAC photometry, for the objects matched with our optical catalog. The dashed line denotes the area where Class II objects are located, based on Allen et al. (2004). Open circles mark the collection of YSOs and pre-main-sequence stars from Merín et al. (2008). Squares mark the objects whose spectra were obtained as part of the SONYC follow-up, and the red stars mark the three confirmed VLMOs.

(A color version of this figure is available in the online journal.)

(VMMPS). VMMPS allows for only two priority classes. In P89 the high-priority list included the two proper motion selected samples, thus containing 59 candidates from the " $I J$-pm" sample and 19 candidates from the "IRAC-pm" sample, while the rest were the photometrically selected candidates from $I J$ and Spitzer-IRAC lists. In P87, prior to our proper motion analysis, the high-priority candidates were those from the selection box, and the rest were randomly chosen objects within the FOV. In total, we obtained spectra of 123 objects from the selection box and 44 outside of it. Of the 123 spectra from the $I J$ selection box, 32 are from the " $I J$-pm" sample. Diamonds in Figure 2 mark all the candidates with spectroscopy inside our selection box.

\subsection{Preliminary Selection}

Our goal is to identify young, low-mass stellar and substellar members of Lupus 3. Among the photometrically selected candidates, we may find contaminants such as embedded stellar members of Lupus 3 with higher masses, reddened background M-type stars, and (less likely) late M-type and early L-type objects in the foreground. The red portion of M-dwarf optical spectra is dominated by molecular features (mainly $\mathrm{TiO}$ and VO; Kirkpatrick et al. 1991, 1995), which allows a relatively simple preliminary selection of candidates based on visual inspection. Here we included all the objects with clear evidence but also, for the sake of caution, those objects with only tentative evidence of molecular features. Another property that was used in the selection is the slope, which should be positive in the red portion of the optical regime for M-type stars. Earlier stellar types typically have negative slopes or appear flat (late K-type). Among the rejected spectra, we mainly find flat spectra with no or with very weak indication of molecular features (late K-dwarfs or reddened earlier-type stars) and reddened featureless spectra (background or embedded stars of spectral type earlier than $\mathrm{K}$ ). We also looked for the $\mathrm{H}_{\alpha}$ emission at $6563 \AA$; only in one case an object was selected solely on the basis of showing $\mathrm{H}_{\alpha}$ and despite the spectrum showing no prominent features characteristic for the M-type stars. After this preliminary selection, our sample contains 27 spectra; 26 of the selected spectra are photometric candidates, while 1 source appears bluer than expected for Lupus members. This source, SONYC-Lup3-6, will be discussed in more detail in Section 4.8.

\subsection{Spectral Features as Membership Indicators}

VIMOS was used in the spectroscopic part of the SONYC campaign in Cha-I (Mužić et al. 2011). The analysis presented here is partly similar to the one in that paper. Since the Cha-I observations in the first half of 2009, VIMOS was equipped with new, more red-sensitive detectors, which substantially decreased fringing that previously contaminated spectra longward of $8000 \AA$. This is fortunate not only because our wavelength range is increased but also because it allows us to analyze the gravity-sensitive Na I features at $\sim 8200 \AA$, which can provide an important constraint for the age of each observed object.

Youth provides strong evidence for cluster membership. We use the following features to identify members of Lupus 3:

1. The Na I doublet at $8183 / 8195 \AA$, which shows a change in EWs, and a strong increase in the depth of absorption with increasing the surface gravity (e.g., Martín et al. 1999; Riddick et al. 2007; Schlieder et al. 2012). Although the individual lines of the doublet cannot be resolved in our spectra, the overall strength of the feature can still be used to assess the membership in the cluster even at low spectral resolutions.

2. The spectral region $\sim 6900-7100 \AA$, shaped by $\mathrm{TiO}$ and $\mathrm{CaH}$ bands, also strongly affected by gravity at $\mathrm{M}$ types (Kirkpatrick et al. 1991). In particular, $\mathrm{CaH}$ absorption shortward of $7050 \AA$ is stronger in M-type dwarfs, making the peak of the feature appear sharper in dwarfs and rather blunt in giants.

3. $\mathrm{H}_{\alpha}$ emission, which is usually interpreted as a sign of accretion, and thus can be used as additional evidence for youth.

4. The Ca II triplet lines in absorption at $8498 \AA, 8542 \AA$, and $8662 \AA$ are prominent in late-K through mid-M giants and are weaker in dwarfs (Kirkpatrick et al. 1991). This feature can help in identifying giant contaminants at early$\mathrm{M}$ spectral types where the Na I doublet becomes of limited use.

The points (1), (2), and (4) are demonstrated in Figure 6, showing a part of the spectral sequence (M1 to M9 with a step of two spectral subtypes) of giants, dwarfs, and members of young star-forming regions (see Section 4.3 for detailed information on spectra and citations). The sodium absorption is much stronger in field dwarfs when compared with lowgravity atmospheres and thus can be used to discard background field dwarfs with SpT later than M4 from our sample. Also, in the low-resolution spectra of dwarfs, the absorption feature is deepest at about the average wavelength of the Na I doublet (vertical line in Figure 6) and appears symmetric around it. On the other hand, the feature appears asymmetric in giants with the minimum slightly shifted toward longer wavelengths. This property can be used to distinguish between giants and low-gravity young objects at spectral types later than $\sim \mathrm{M} 6$. Determination of the spectral class is less straightforward at 


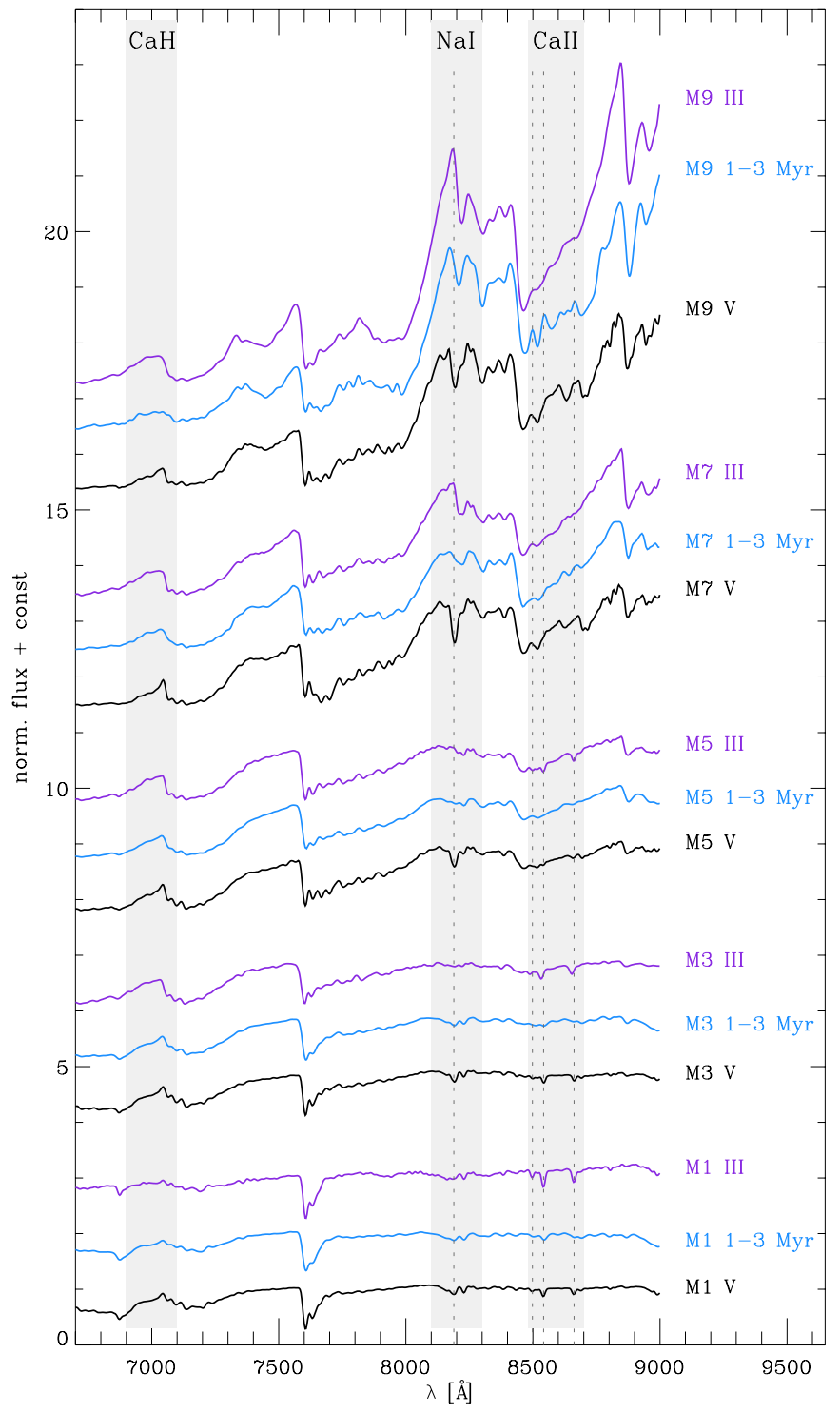

Figure 6. Part of the spectral sequence used for the spectral type fitting. Templates include dwarf and giant stars with spectral type M1-M9, as well as the young members of star-forming regions. Three gravity-sensitive spectral regions are shaded: (1) the $7000 \AA$ region affected by $\mathrm{CaH}$ absorption, (2) the region around the $\mathrm{NaI}$ absorption feature at $\sim 8200 \AA$, and (3) the Ca II absorption triplet at $\sim 8600 \AA$. The average wavelength of the Na I doublet and the Ca II triplet are marked by the vertical dashed lines. The spectra have been smoothed to match the spectral resolution of VIMOS and are normalized at $7500 \AA$ A.

(A color version of this figure is available in the online journal.)

early spectral types (M2-M3), where the $\mathrm{CaH}$ feature at $7000 \AA$ and the Ca II triplet can provide additional help. At M1 and earlier, the low-resolution spectra start to be of limited value for membership determination.

The equivalent width (EW) of the $\mathrm{H}_{\alpha}$ line in emission is often used to distinguish the accreting objects from the nonaccretors. While the chromospheric $\mathrm{H}_{\alpha}$ emission is expected to be found in most pre-main-sequence M-dwarfs because of magnetic activity, larger EWs observed in Class II objects are usually interpreted as a consequence of accretion and/or outflows (e.g., Scholz et al. 2007; Stelzer et al. 2013). White \& Basri (2003) propose the $\mathrm{EW}\left(\mathrm{H}_{\alpha}\right)$ for actively accreting stars to be larger than $3 \AA$ for K0-K5 stars, > $10 \AA$ for K7-M2.5, $>20 \AA$ for M3-M5.5, and > $40 \AA$ for M6-M7.5 stars. A similar criterion, but with finer division with respect to spectral type and extended to the substellar regime, was derived by Barrado y Navascués \& Martín (2003). The $\operatorname{EW}\left(\mathrm{H}_{\alpha}\right)$ for the SONYC objects in Lupus 3 are given in Table 2. SONYC-Lup3-1, -7, and -10 are revealed as strong accretors, and SONYC-Lup3-2, -8 , and -11 are clearly non-accretors. The remaining objects with measured $\mathrm{EW}\left(\mathrm{H}_{\alpha}\right)$ all appear to be undergoing accretion according to the Barrado y Navascués \& Martín (2003) criterion, whereas SONYC-Lup3-5, -6, and -12 fall slightly below the thresholds set by White \& Basri (2003) for their respective spectral types.

\subsection{Spectral Classification}

To determine spectral type and extinction of selected candidate members, we compare each spectrum with an empirical grid consisting of spectral templates of field dwarfs, giants, and members of young star-forming regions with spectral types M1 to M9. A grid of field dwarfs separated by the 0.5 spectral subtype was created by averaging a number of available spectra at each sub-type. In the case of giants, the grid consists of a collection of available spectra, with a step of $0.5-1$ spectral subtypes. ${ }^{11}$ The grid of young objects (1-3 Myr) consists of the spectra of objects in Cha I, Taurus, and $\eta$ Cha (Luhman et al. 2003a; Luhman 2004a, 2004b, 2004c), with steps of 0.25-0.5 spectral subtypes.

Spectral type and extinction can be fitted simultaneously. The template spectra were first smoothed to match the resolution of the VIMOS spectra and rebinned to the same wavelength grid. The region around the $\mathrm{H}_{\alpha}$ line was masked. The best-fit parameters were determined by minimizing the test value $\chi$ defined as

$$
\chi=\frac{1}{N} \sum_{i=1}^{N} \frac{(O-T)^{2}}{T},
$$

where $O$ is the object spectrum, $T$ the template spectrum, and $N$ the number of data points.

As mentioned before, spectral classification becomes less reliable at low resolution for objects of spectral type M3 and earlier. It is therefore not surprising that at these early spectral types, the fitting scheme gives similar results for different spectral classes. Some level of degeneracy with respect to spectral classes is also present at later spectral types, but only in a few cases. It is therefore clear that the final assessment of class (i.e., membership) cannot be based on this fitting scheme only. However, there is much less degeneracy present with respect to the spectral subtype and $A_{V}$, even for the objects with degenerate spectral classes. Typically, several best-fit results cluster within \pm 1 spectral subtype and \pm 1 mag in $A_{V}$, or less, from the best-fit value. We therefore adopt these values as typical uncertainties of the fitting procedure.

To determine the membership status of an object, several best fits at each spectral class are inspected visually in order to check for the presence or shape of the spectral features discussed in the previous section.

Of the initial 27 spectra we started with, 9 objects are classified as field dwarfs because of the deep Na I absorption and the lack of $\mathrm{H}_{\alpha}$ emission (see Figure 7). Two spectra are best represented by spectra of early-M giants, while four objects have M-dwarf features that are less pronounced than in M1 dwarfs,

\footnotetext{
11 Spectra of field dwarfs and giants are available from http://www.dwarfarchives.org, http://kellecruz.com/M_standards/ and from Luhman et al. (2003a), and Luhman (2004c).
} 
Table 2

Photometry and Spectral Types for the Objects of Spectral Type M, or Slightly Earlier, Identified Toward Lupus 3

\begin{tabular}{|c|c|c|c|c|c|c|c|c|c|c|c|c|c|c|}
\hline ID & $\alpha(\mathrm{J} 2000)$ & $\delta(\mathrm{J} 2000)$ & $\begin{array}{c}i \\
(\mathrm{mag})\end{array}$ & $\begin{array}{c}J \\
(\mathrm{mag})\end{array}$ & $\begin{array}{c}K \\
(\mathrm{mag})\end{array}$ & $\begin{array}{l}A_{V}^{\text {phot }} \\
(\mathrm{mag})\end{array}$ & $\mathrm{SpT}$ & $\begin{array}{l}A_{V}^{\mathrm{SpT}} \\
(\mathrm{mag})\end{array}$ & $\begin{array}{c}\mu_{\alpha} \cos \delta \\
\left(\mathrm{mas} \mathrm{yr}^{-1}\right)\end{array}$ & $\begin{array}{c}\mu_{\delta} \\
\left(\operatorname{mas~yr}^{-1}\right)\end{array}$ & $\begin{array}{c}\mu- \\
\text { cand? }\end{array}$ & $\begin{array}{c}\mathrm{EW}(\mathrm{H} \alpha) \\
(\AA)\end{array}$ & Membership & Comments \\
\hline S-1 & $16: 07: 08.55$ & $-39: 14: 07.8$ & 17.3 & 14.6 & 11.5 & 11.5 & M 1.75 & 3.0 & $-4.2 \pm 5.2$ & $-21.2 \pm 4.7$ & $\mathrm{y}$ & -77.6 & Lupus 3 & M08 \\
\hline S-2 & $16: 07: 33.82$ & $-39: 03: 26.5$ & 19.4 & 16.2 & 14.1 & 6.0 & M 3.00 & 5.0 & $0.4 \pm 7.1$ & $-16.3 \pm 5.9$ & $\mathrm{y}$ & -4.9 & uncertain & \\
\hline S-3 & $16: 07: 55.18$ & $-39: 06: 04.0$ & 16.2 & 13.5 & 11.4 & 5.9 & M 5.75 & 2.0 & $-12.4 \pm 7.1$ & $-22.8 \pm 5.9$ & $\mathrm{y}$ & -37.2 & Lupus 3 & $\mathrm{M} 6.5^{\mathrm{d}}, \mathrm{C} 09, \mathrm{G} 06$ \\
\hline S-4 & 16:08:04.76 & $-39: 04: 49.6$ & 17.0 & 13.9 & 12.4 & 2.9 & M 6.50 & 1.0 & $-8.1 \pm 7.1$ & $-22.6 \pm 5.9$ & $\mathrm{y}$ & -44.4 & Lupus 3 & $\mathrm{M} 7^{\mathrm{d}}, \mathrm{C} 09$ \\
\hline S-5 & $16: 08: 16.03$ & $-39: 03: 04.5$ & 15.5 & 12.5 & 11.1 & 2.5 & M 6.25 & 1.0 & $-9.3 \pm 7.1$ & $-19.9 \pm 5.9$ & $\mathrm{y}$ & -37.5 & Lupus 3 & Par-Lup3-1; M7.5 ${ }^{\mathrm{a}}, \mathrm{M} 5.5^{\mathrm{b}}$ \\
\hline S-6 & 16:08:59.52 & $-38: 56: 20.8$ & 19.1 & 17.4 & 16.7 & 0.0 & M 4.75 & 0.0 & $-6.5 \pm 7.1$ & $-19.4 \pm 5.9$ & $\mathrm{y}$ & -18.6 & uncertain & \\
\hline S-7 & 16:08:59.53 & $-38: 56: 27.8$ & 17.1 & 13.9 & 12.7 & 1.3 & M 8.00 & 0.5 & $-8.8 \pm 7.1$ & $-19.6 \pm 5.9$ & $\mathrm{y}$ & -185.7 & Lupus 3 & M8c, G06 \\
\hline S-8 & $16: 10: 18.13$ & $-39: 10: 33.6$ & 19.8 & 16.2 & 13.8 & 7.3 & M 1.00 & 6.5 & $-16.2 \pm 5.3$ & $-9.8 \pm 5.0$ & $\mathrm{y}$ & -4.6 & uncertain & \\
\hline S-9 & $16: 11: 26.34$ & $-39: 02: 17.1$ & 19.3 & 16.2 & 14.0 & 6.4 & M 1.00 & 5.5 & $-14.6 \pm 5.3$ & $-11.3 \pm 5.0$ & $\mathrm{y}$ & $\ldots$ & uncertain & \\
\hline S-10 & 16:09:13.43 & $-38: 58: 04.9$ & 19.5 & 16.1 & 14.9 & 1.5 & M 8.75 & 0.0 & $-13.8 \pm 7.1$ & $-20.9 \pm 5.9$ & $\mathrm{y}$ & -48.8 & Lupus 3 & \\
\hline S-11 & 16:12:05.91 & $-39: 04: 07.5$ & 18.7 & 15.7 & 14.4 & 1.5 & M 3.00 & 3.0 & $-24.7 \pm 5.3$ & $-20.5 \pm 5.0$ & $\mathrm{y}$ & -5.4 & uncertain & \\
\hline S-12 & $16: 11: 18.47$ & $-39: 02: 58.2$ & 18.0 & 15.1 & 13.3 & 4.2 & $<\mathrm{M} 1$ & $\ldots$ & $-6.1 \pm 5.3$ & $-8.5 \pm 5.0$ & $\mathrm{y}$ & -12.1 & Lupus 3 & \\
\hline S-13 & $16: 07: 36.48$ & $-39: 04: 53.8$ & 15.7 & 12.9 & 10.6 & 7.1 & M 0.00 & 3.5 & $-3.4 \pm 7.1$ & $1.1 \pm 5.9$ & $\mathrm{n}$ & $\ldots$ & $\mathrm{III}^{2}$ & \\
\hline S-14 & 16:08:01.42 & $-39: 10: 16.1$ & 19.9 & 16.4 & 14.0 & 7.7 & $<\mathrm{M} 1$ & $\ldots$ & $-8.4 \pm 7.1$ & $-12.7 \pm 5.9$ & $\mathrm{y}$ & $\ldots$ & uncertain & \\
\hline S-15 & $16: 07: 52.46$ & $-39: 09: 48.1$ & 19.0 & 15.6 & 13.2 & 7.6 & M 2.50 & 4.7 & $-29.8 \pm 7.1$ & $-23.1 \pm 5.9$ & $\mathrm{y}$ & $\ldots$ & $\mathrm{V}^{3}$ & \\
\hline S-16 & 16:10:14.95 & $-39: 10: 16.2$ & 19.9 & 16.4 & 14.3 & 5.7 & M 4.50 & 4.7 & $5.9 \pm 5.3$ & $24.0 \pm 5.0$ & $\mathrm{n}$ & $\ldots$ & V & \\
\hline S-17 & 16:11:56.11 & $-39: 08: 23.8$ & 15.3 & 12.8 & 11.1 & 4.2 & $<\mathrm{M} 1$ & $\ldots$ & $-0.3 \pm 5.3$ & $11.1 \pm 5.0$ & $\mathrm{n}$ & $\ldots$ & III & \\
\hline S-18 & 16:11:09.03 & $-39: 06: 22.7$ & 16.8 & 14.3 & 12.5 & 4.5 & M 2.50 & 3.0 & $-11.4 \pm 5.3$ & $-21.7 \pm 5.0$ & $\mathrm{y}$ & $\ldots$ & $\mathrm{V}$ & \\
\hline S-19 & $16: 11: 39.24$ & $-39: 06: 32.8$ & 15.4 & 12.5 & 10.4 & 6.0 & M 0.50 & 3.0 & $-5.7 \pm 5.3$ & $-5.4 \pm 5.0$ & $\mathrm{y}$ & $\ldots$ & III & \\
\hline S-20 & $16: 11: 36.20$ & $-39: 02: 36.9$ & 20.4 & 16.8 & 14.6 & 6.9 & M 4.00 & 5.3 & $3.9 \pm 5.3$ & $17.1 \pm 5.0$ & $\mathrm{n}$ & $\ldots$ & $\mathrm{V}$ & \\
\hline S-21 & $16: 11: 33.34$ & $-38: 59: 44.3$ & 19.9 & 16.5 & 14.3 & 4.9 & M 3.00 & 4.5 & $7.8 \pm 5.3$ & $1.1 \pm 5.0$ & $\mathrm{n}$ & $\ldots$ & $\mathrm{V}$ & \\
\hline S-22 & 16:10:47.49 & $-38: 57: 12.4$ & 20.4 & 17.1 & 16.2 & 0.0 & $<\mathrm{M} 1$ & $\ldots$ & $\ldots$ & $\ldots$ & $\ldots$ & $\ldots$ & uncertain & \\
\hline S-23 & $16: 10: 45.40$ & $-38: 54: 54.9$ & 16.1 & 14.3 & 12.9 & 2.1 & $<\mathrm{M} 1$ & $\ldots$ & $-7.9 \pm 5.3$ & $-1.9 \pm 5.0$ & $\mathrm{n}$ & $\ldots$ & III & M08, C09 \\
\hline S-24 & 16:07:09.06 & $-39: 01: 07.6$ & 19.8 & 18.1 & 16.6 & 2.8 & M 1.50 & 1.5 & $7.4 \pm 5.2$ & $3.6 \pm 4.7$ & $\mathrm{n}$ & $\ldots$ & $\mathrm{V}$ & \\
\hline S-25 & 16:12:04.96 & $-39: 06: 11.4$ & 19.9 & 17.9 & 16.0 & 5.1 & M 2.50 & 3.0 & $1.0 \pm 5.3$ & $2.7 \pm 5.0$ & $\mathrm{n}$ & $\ldots$ & $\mathrm{V}$ & \\
\hline S-26 & $16: 10: 56.40$ & $-39: 04: 31.7$ & 19.1 & 17.3 & 15.7 & 3.4 & M 5.0 & 2.5 & $35.4 \pm 5.3$ & $0.9 \pm 5.0$ & $\mathrm{n}$ & $\ldots$ & $\mathrm{V}$ & \\
\hline S-27 & $16: 11: 15.08$ & $-39: 07: 15.0$ & 18.1 & 16.4 & 14.7 & 3.6 & M 7.0 & 0.0 & $\ldots$ & $\ldots$ & $\ldots$ & $\ldots$ & $\mathrm{V}$ & \\
\hline
\end{tabular}

Notes.

The IDs SONYC-Lup3-X are abbreviated with S-X.

${ }^{2}$ Background giant.

${ }^{3}$ Field dwarf.

Proper motion candidate.

${ }^{5}$ C09—candidate member in Comerón et al. (2009), Table 9; M08-IRAC YSO candidate from Merín et al. (2008) G06-X-ray source from Gondoin (2006).

References. (a) Comerón et al. 2003; (b) Mortier et al. 2011; (c) Allen et al. 2007; (d) Comerón et al. 2013. 


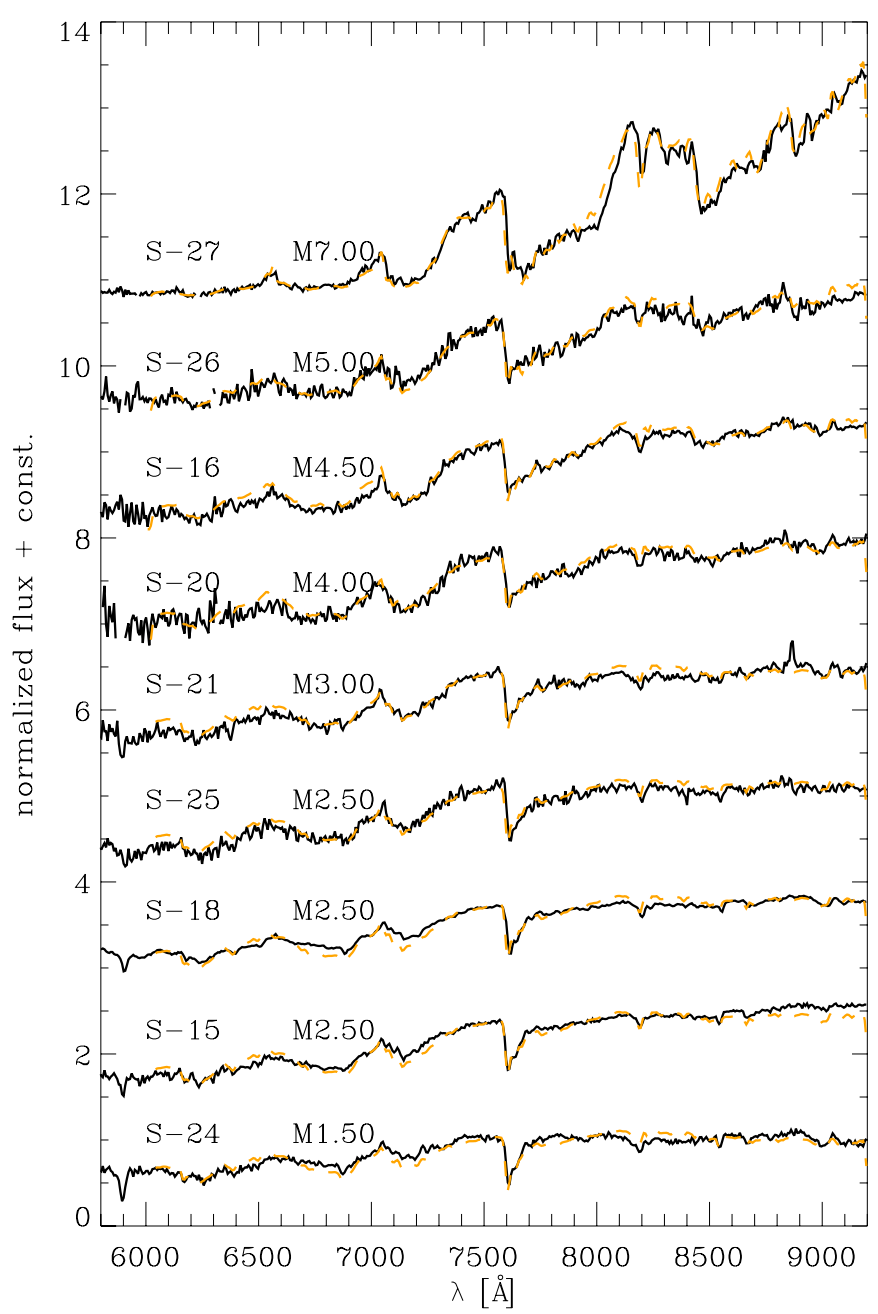

Figure 7. Spectra of the objects identified as field dwarfs (Table 2; black), along with the best-fit spectra (orange dashed line). The spectral resolution of the fit templates has been reduced in order to match the resolution of our spectra. Each spectrum has been corrected for extinction (see Table 2) but not for the atmospheric absorption.

(A color version of this figure is available in the online journal.)

i.e., are probably of earlier spectral type (bottom 6 spectra in Figure 9). The spectra of the latter four objects (SONYC-Lup3$14,-17,-22$, and -23) all show evidence for the Ca II triplet in absorption. The spectra of SONYC-Lup3-17 and -23 also have very weak or non-existent $\mathrm{NaI}$ absorption and thus are probably background giants. The spectra of SONYC-Lup3-14 and -22 have lower signal-to-noise ratio, which poses a difficulty in final classification. The depth of the $\mathrm{Na}$ I in the spectrum of SONYC-Lup3-14 hints to a high-gravity atmosphere, but for the sake of caution, we prefer to keep the membership status of both objects as uncertain.

Seven spectra are best fit by young dwarf templates with no degeneracy in spectral class (SONYC-Lup3-1, -3,- 4, -5, -6, -7, and -10). The spectrum of the object SONYC-Lup3-10 is best matched with a M8.75 young dwarf template, which was derived by averaging the spectra of Taurus members KPNO09 (M8.5) and KPNO12 (M9) from Luhman (2004c). All of these seven objects also show $\mathrm{H}_{\alpha}$ emission and appear to have weak $\mathrm{Na}$ I absorption, suggesting some kind of low-gravity atmosphere. All seven objects are also proper motion candidates. SONYCLup3-12 exhibits strong $\mathrm{H}_{\alpha}$ emission, but its spectrum is outside our fitting grid (earlier than M1). The shape of the Na I feature

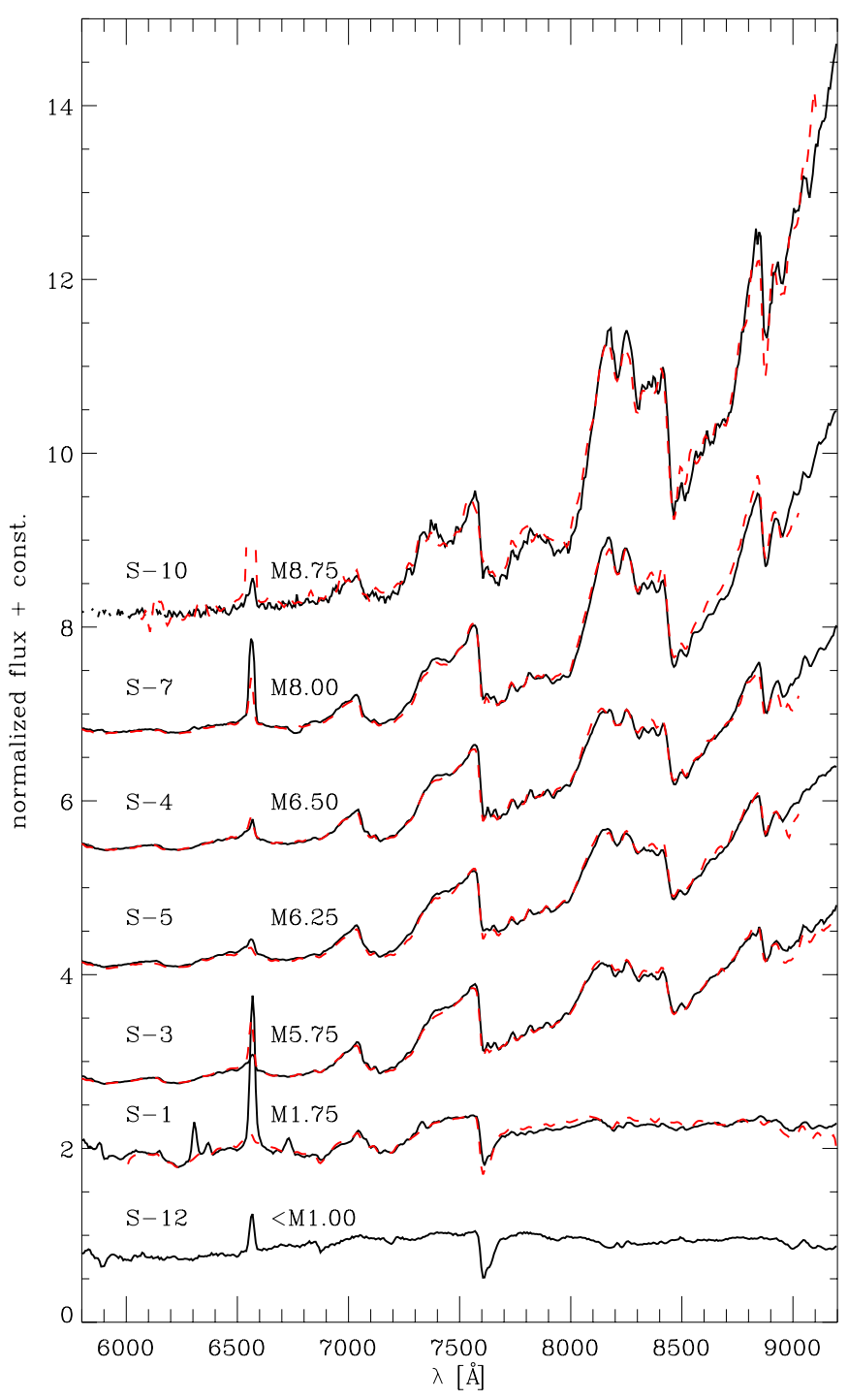

Figure 8. Spectra of the objects identified as probable members of Lupus 3 (Table 2; black), along with the best-fit spectra of members of young starforming regions (red dashed line). The spectral resolution of the fit templates has been reduced in order to match the resolution of our spectra. Each spectrum has been corrected for extinction (see Table 2) but not for the atmospheric absorption.

(A color version of this figure is available in the online journal.)

reveals a low-gravity atmosphere, while the lack of the Ca II triplet in absorption makes it less probable to be a giant. Furthermore, its proper motion argues in favor of its membership in Lupus, and therefore we include SONYC-Lup3-12 in the list of candidate members. The spectra of these eight best candidates for membership in Lupus are shown in Figure 8, with the exception of SONYC-Lup3-6, which is, for the reasons that will become apparent in the next sections, classified as uncertain and shown in Figure 9.

The nature of the remaining four objects of early $\mathrm{M}$ spectral type (SONYC-Lup3-2,-8, -9, and -11) remains uncertain at this stage. In Figure 9 we show their spectra together with the bestfit young dwarf templates, but we note that other classes might give an equally satisfactory fit. The $\mathrm{CaH}$ and $\mathrm{Na}$ I regions for all 4 objects fit better in the dwarf class rather than giants. The Ca II triplet absorption is present, which indicates that none of these objects are young. 


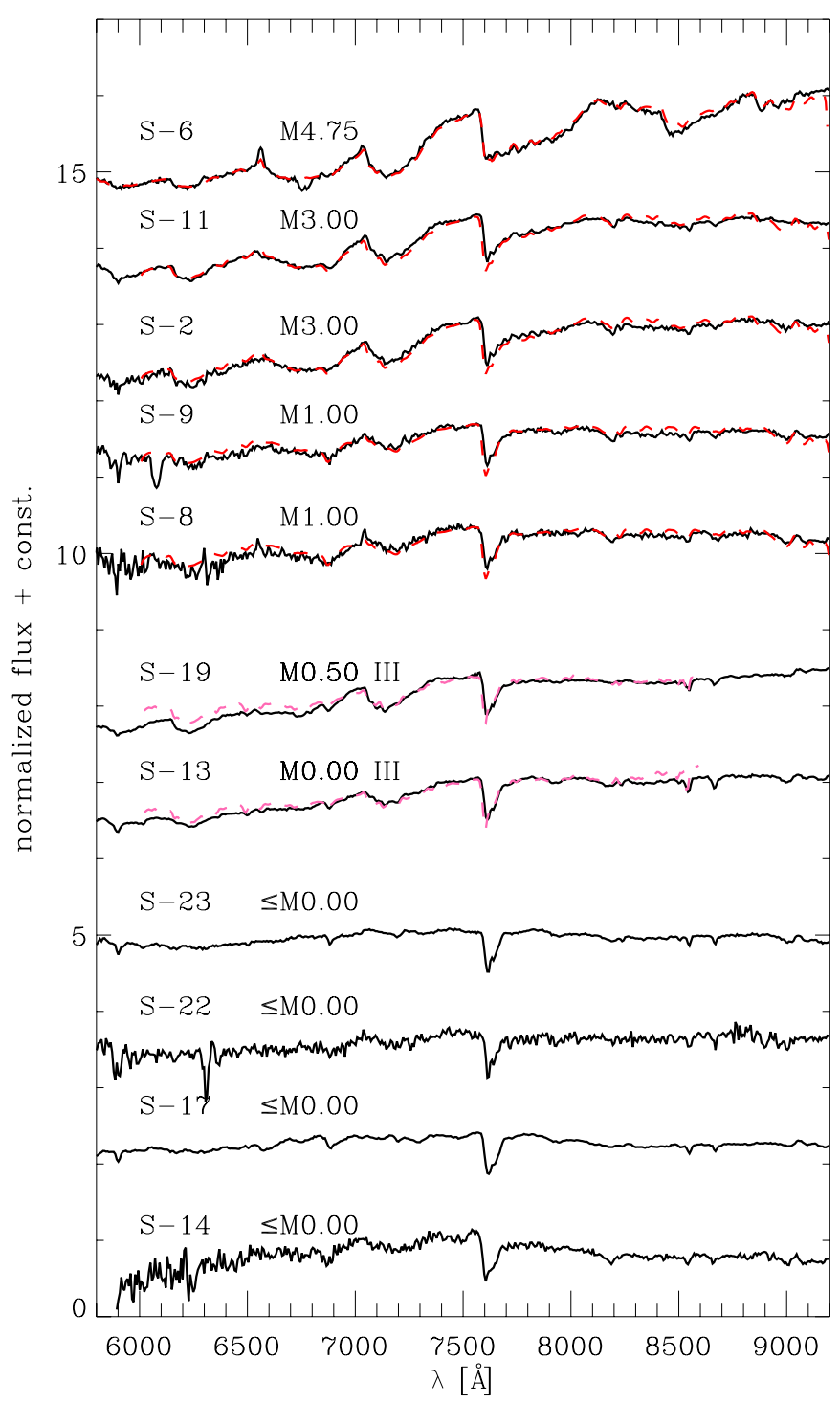

Figure 9. Spectra of the objects identified as giants, or with uncertain classification (Table 2; black), along with the best-fit spectra (dashed colored line). The spectral resolution of the fit templates has been reduced in order to match the resolution of our spectra. Each spectrum has been corrected for extinction (see Table 2) but not for the atmospheric absorption.

(A color version of this figure is available in the online journal.)

\subsection{Model Fitting}

In order to derive effective temperatures for the SONYC objects, we perform the spectral model fitting using the BT-Settl (Allard et al. 2011) and AMES-Dusty (Allard et al. 2001) models. Model spectra are smoothed by a boxcar function beforehand to match the wavelength step of the VIMOS spectra. The three main parameters that affect the spectral shape are the effective temperature $\left(T_{\text {eff }}\right)$, gravity $(\log g)$, and interstellar extinction $\left(A_{V}\right)$. Fitting the models to our low-resolution spectra does not allow an unambiguous determination of all the three parameters at the same time. We therefore keep $\log g$ constant for each object and assume the value of 3.5 (or 4 in case of AMES-Dusty models above $3000 \mathrm{~K}$ ) for the objects identified as probable young dwarfs, 5 for the field dwarfs, and 3 for the giants (in this case only BT-Settl models are used). For all the spectra classified as uncertain, we use the intermediate value of $\log g$, i.e., the same as for young dwarfs. The fitting procedure
Table 3

Effective Temperature and Extinction Derived from Models, for the Objects of Spectral Type M (or Slightly Earlier) Toward Lupus 3

\begin{tabular}{lcccccc}
\hline \hline ID & $\begin{array}{c}T_{\text {eff }}^{A-D} \\
(\mathrm{~K})\end{array}$ & $\begin{array}{c}A_{V}^{A-D} \\
(\mathrm{mag})\end{array}$ & $\begin{array}{c}T_{\text {eff }}^{B T-S} \\
(\mathrm{~K})\end{array}$ & $\begin{array}{c}A_{V}^{B T-S} \\
(\mathrm{mag})\end{array}$ & $T_{\text {eff }}^{C}$ & $A_{V}^{C}$ \\
\hline S-1 & 3900 & 3.0 & 3800 & 3.0 & $4400^{\mathrm{a}}$ & 7.1 \\
S-2 & 3600 & 6.0 & 3500 & 5.0 & $\ldots$ & $\ldots$ \\
S-3 & 3100 & 4.0 & 3000 & 3.0 & $2950^{\mathrm{b}}$ & 2.3 \\
S-4 & 3000 & 4.0 & 3000 & 3.5 & $2900^{\mathrm{b}}$ & 3.5 \\
S-5 & 3000 & 3.5 & 2900 & 2.0 & $2800^{\mathrm{a}}$ & 2.0 \\
S-6 & 3100 & 0.5 & 3200 & 0.0 & $\ldots$ & $\ldots$ \\
S-7 & 2900 & 4.5 & 2800 & 3.0 & $2600^{\mathrm{a}}$ & 1.5 \\
S-8 & 3900 & 6.5 & 3800 & 6.5 & $\ldots$ & $\ldots$ \\
S-9 & 3900 & 5.5 & 3800 & 5.5 & $\ldots$ & $\ldots$ \\
S-10 & 2600 & 4.0 & 2700 & 4.0 & $\ldots$ & $\ldots$ \\
S-11 & 3700 & 4.0 & 3500 & 3.5 & $\ldots$ & $\ldots$ \\
S-12 & $>3900$ & $\ldots$ & 4200 & 4.5 & $\ldots$ & $\ldots$ \\
S-13 & $\ldots$ & $\ldots$ & 3900 & 4.5 & $\ldots$ & $\ldots$ \\
S-14 & 3900 & 6.0 & 3800 & 6.5 & $\ldots$ & $\ldots$ \\
S-15 & 3700 & 6.5 & 3600 & 6.0 & $\ldots$ & $\ldots$ \\
S-16 & 3100 & 5.5 & 3000 & 4.0 & $\ldots$ & $\ldots$ \\
S-17 & $\ldots$ & $\ldots$ & 4400 & 5.0 & $\ldots$ & $\ldots$ \\
S-18 & 3800 & 4.5 & 3600 & 3.5 & $\ldots$ & $\ldots$ \\
S-19 & $\ldots$ & $\ldots$ & 3900 & 4.0 & $\ldots$ & $\ldots$ \\
S-20 & 3400 & 6.5 & 3200 & 5.5 & $\ldots$ & $\ldots$ \\
S-21 & 3400 & 5.0 & 3300 & 4.5 & $\ldots$ & $\ldots$ \\
S-22 & $>4000$ & $\ldots$ & 4100 & 2.5 & $\ldots$ & $\ldots$ \\
S-23 & $\ldots$ & $\ldots$ & 4200 & 2.5 & $4400^{\mathrm{a}}$ & 2.7 \\
S-24 & 3900 & 2.0 & 3800 & 2.0 & $\ldots$ & $\ldots$ \\
S-25 & 3400 & 3.5 & 3300 & 3.0 & $\ldots$ & $\ldots$ \\
S-26 & 3100 & 4.0 & 3000 & 2.5 & $\ldots$ & $\ldots$ \\
S-27 & 2700 & 2.5 & 2800 & 1.5 & $\ldots$ & $\ldots$ \\
\hline
\end{tabular}

Notes. A-D-AMES-Dusty (Allard et al. 2001); BT-S—BT-Settl (Allard et al. 2011); C-parameters derived by (a) Comerón et al. (2009) and (b) Comerón et al. (2013).

of $T_{\text {eff }}$ and $A_{V}$ is identical to the one described in Mužić et al. (2011); $T_{\text {eff }}$ is varied between $2000 \mathrm{~K}$ and $4500 \mathrm{~K}$ in steps of $100 \mathrm{~K}$, and $A_{V}$ is varied from 0 to 10 in steps of $0.5 \mathrm{mag}$. To de-redden our spectra, we apply the extinction law from Cardelli et al. (1989), assuming $R_{V}=4$.

The spectra of objects in Table 2 with the best-fit atmosphere models are shown in the appendix (Figures 14 and 15), and the results of our spectral fitting procedure for these objects are given in Table 3 . The uncertainty is $100 \mathrm{~K}$ for the derived effective temperature, and $0.5 \mathrm{mag}$ for the extinction, which reflects the spacing of the grid.

\subsection{MIR-excess}

From the list of potential members selected from the IRAC data, we obtained spectra of 11 objects. Three of them are confirmed as VLM members of Lupus 3 (SONYC-Lup3-1, SONYC-Lup3-7, and SONYC-Lup3-10). Of the confirmed objects, all were originally selected from the iJ selection. SONYC-Lup3-1 satisfies the YSO criteria from Merín et al. (2008). The fact that these three candidates exhibit disk excess further strengthens their membership in Lupus 3.

There is only one object in the Spitzer photometric candidate selection (Figure 5) that appears as a candidate YSO in Merín et al. (2008), but it was not confirmed in our work. Judging from the spectrum, this object (c2d source number $120 ; \alpha=$ $16: 10: 45.40, \delta=-38: 54: 54.9$ ) probably belongs to the spectral type $\mathrm{K}$, i.e., it is too early for our spectral typing scheme to work. Therefore, while this object does not fall into the 


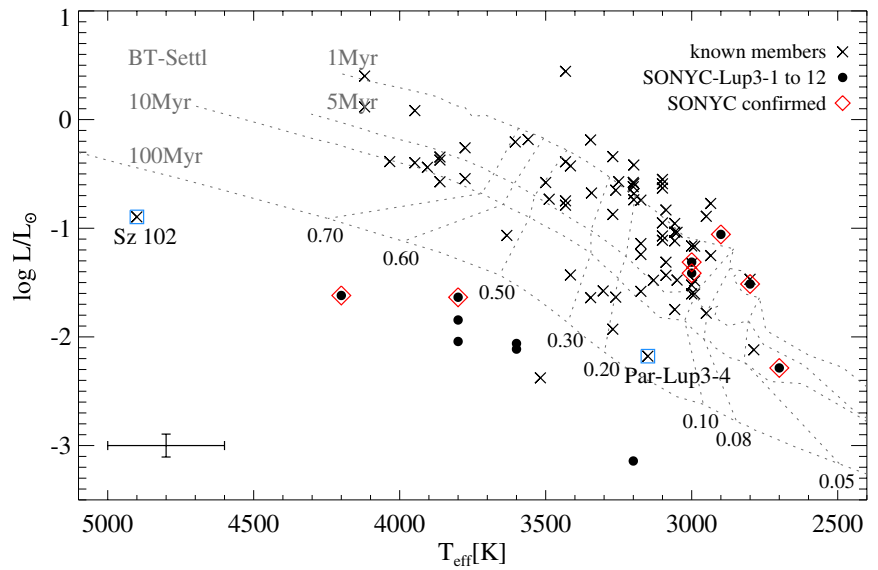

Figure 10. H-R diagram for the Lupus 3 members with existing $T_{\text {eff }}$ or spectral type from the literature (crosses). Filled circles mark the sources SONYCLup3-1 to -12. Red diamonds mark the final subset of confirmed members of Lupus 3. The two well-known examples of underluminous, emission line YSOs are labeled and marked with blue squares. The dashed lines show BT-Settl theoretical tracks for several ages spanning 1 to $100 \mathrm{Myr}$.

(A color version of this figure is available in the online journal.)

class of VLMOs that are the subject of this paper, we do not rule it out as a potential member. The remaining spectra also look earlier than M1 and do not show any evidence of $\mathrm{H}_{\alpha}$ emission; therefore, they are eliminated in our search for VLM objects.

\subsection{Hertzsprung-Russell Diagram}

In Figure 10 we show the Hertzsprung-Russell (H-R) diagram for the list of spectroscopically confirmed members shown in Table 4 . The extinction was calculated by assuming the intrinsic color $(J-K)_{0}=1$ and extinction law from Cardelli et al. (1989) with $R_{V}=4 .^{12}$ The adopted distance is $200 \mathrm{pc}$. Where available, we use $T_{\text {eff }}$ estimates available from the literature. For the spectroscopically confirmed members that lack the $T_{\text {eff }}$ information, we convert their spectral types to $T_{\text {eff }}$ using the transformation established in Section 5.1. We calculate the bolometric correction in the $J$ band $\left(\mathrm{BC}_{J}\right)$ from the polynomial relation between $\mathrm{BC}_{J}$ and $T_{\text {eff }}$ derived by Pecaut \& Mamajek (2013) for pre-main-sequence stars with ages of 5-30 Myr. The typical error bar is shown in the lower left corner. The uncertainty in the $T_{\text {eff }}$ is set to $200 \mathrm{~K}(\sim 200 \mathrm{~K}$ for sources from Comerón et al. 2009, $300 \mathrm{~K}$ for Mortier et al. 2011, and $\sim 100 \mathrm{~K}$ for the rest). The error in luminosity takes into account the typical error in the observed $J$ magnitude $(0.1 \mathrm{mag})$, distance $( \pm 20 \mathrm{pc}), A_{V}( \pm 1 \mathrm{mag})$, and $\mathrm{BC}_{J}$. We also show the BT-Settl isochrones for ages between $1 \mathrm{Myr}$ and $100 \mathrm{Myr}$. Filled circles mark the 8 objects that were identified as possible members in Section 4.3, together with the 4 objects with uncertain membership status (SONYC-Lup3-1 through -12). We use $T_{\text {eff }}$ and $A_{V}$ as derived by comparison with the BT-Settl models.

The position of sources in $\mathrm{H}-\mathrm{R}$ diagram can provide additional information about their nature. We can compare the positions of our sources in the H-R diagram, with the positions of the known members of Lupus 3. The large fraction of previously confirmed

\footnotetext{
12 As argued in Scholz et al. (2009), the value $(J-K)_{0}=1$ is appropriate for objects of the $\mathrm{M}$ spectral type. The only K-type object shown in the H-R diagram is $\mathrm{Sz} 102(\sim \mathrm{K} 2)$, where we adopt a more suitable value of $\sim 0.6$ (Bessell \& Brett 1988). This change in value shifts the point by $\sim 0.3 \mathrm{dex}$ in the positive $y$-direction.
}

members is located between the 1 and 10 Myr isochrones, with a few also found below the $10 \mathrm{Myr}$ isochrone. Among the latter are the two well-known cluster members showing significant underluminosity for their spectral types, Sz 102 and Par-Lup3-4 (Krautter 1986; Comerón et al. 2003). ${ }^{13}$

The H-R diagram positions of the five SONYC sources with estimated $T_{\text {eff }} \leqslant 3000 \mathrm{~K}$ agree well with the positions of other Lupus members. These sources, SONYC-Lup3-3, -4, $-5,-7$, and -10 , are therefore classified as members of Lupus 3. The remaining sources appear well below the $100 \mathrm{Myr}$ BT-Settl isochrone. We compared our photometry with that from 2MASS to check for variability as a possible cause of the underluminosity for some of these seven sources. While SONYC-Lup3-6 is too faint to be detected by 2MASS, all the rest have photometry of a good quality in at least one of the $J$ and $K$ bands. SONYC-Lup3-1 shows a difference of 0.7 mag in the $J$ band and $0.1 \mathrm{mag}$ in the $K$ band; SONYC-Lup3-12 show a difference of $0.3 \mathrm{mag}$ in the $K$ band (only an upper limit in the $J$ band in 2MASS). The remaining sources show no evidence for variability, with differences between the two photometric data sets below $0.1 \mathrm{mag}$, i.e., comparable to the measurement errors. As mentioned in Section 4.3, SONYC-Lup3-2, -8, -9, and -11 were suspected to be non-members, and Figure 10 confirms it. The remaining three sources (SONYC-Lup3-1, -6, and -12) deserve special attention and will be discussed below. Finally, the lack of the objects with similar spectral types (early-tomid M) having luminosities that would suggest membership in Lupus is easily explained by the saturation limit of the $i$-band catalog used for the selection.

\subsection{SONYC-Lup3-1}

In addition to being one of the strongest $\mathrm{H}_{\alpha}$ emitters among our selected sources $\left(\mathrm{EW}\left(\mathrm{H}_{\alpha}\right)=-77.6 \AA\right)$, the spectrum of SONYC-Lup3-1 shows presence of forbidden lines, similar to those previously observed in spectra of Lupus 3 members Par-Lup3-4, Sz102, and Sz106 (Comerón et al. 2003). We identify the [O I] lines at $6300 \AA$ and $6364 \AA$, the [S II] line at $6731 \AA$ (possibly blended with the [S II] line at $6716 \AA$ ), and the [O II] line at $7329 \AA$. Forbidden lines such as those identified here are commonly observed in actively accreting young stars, and their ratios can be used to estimate physical conditions in the circumstellar medium and mass-loss rates (Osterbrock 1989). However, as discussed in Comerón et al. (2003), forbidden lines in T-Tauri spectra are usually split into separate components sampling different physical conditions, which cannot be distinguished with our low-resolution spectra. Moreover, our spectra do not allow definitive identification of some lines (e.g., [S II] at 6731 and $6716 \AA$ ). We therefore refrain from physical interpretation of the conditions causing this emission, and we leave this to future studies at higher spectral resolution.

Two sources in Lupus 3 are reported in the literature to exhibit similar properties. Sz 102 appears underluminous for its spectral type, which was first noted by Hughes et al. (1994). Krautter (1986) found Sz 102 to be associated with a bipolar jet and a Herbig-Haro object. This geometry suggests that we might be viewing the star through a nearly edge-on disk (Krautter 1986; Hughes et al. 1994). Comerón et al. (2003)

\footnotetext{
13 The spectral type of $\mathrm{Sz} 102$ is not well constrained; for the plot we adopt the $T_{\text {eff }}$ of $4900 \mathrm{~K}$ (spectral type K2), according to Mortier et al. (2011). We note that the associated error in $T_{\text {eff }}$ is ${ }_{-840}^{+350} \mathrm{~K}$, i.e., larger than the typical error bar shown in the plot.
} 
Table 4

Spectroscopically Confirmed Very Low Mass Members of Lupus 3

\begin{tabular}{|c|c|c|c|c|c|c|c|}
\hline ID & $\begin{array}{c}\alpha \\
(\mathrm{J} 2000)\end{array}$ & $\begin{array}{c}\delta \\
(\mathrm{J} 2000)\end{array}$ & $\begin{array}{c}J \\
(\mathrm{mag})\end{array}$ & $\begin{array}{c}K \\
(\mathrm{mag})\end{array}$ & $\mathrm{SpT}$ & $\begin{array}{l}T_{\text {eff }} \\
(\mathrm{K})\end{array}$ & Other Names \\
\hline SONYC-Lup3-1 & 160708.55 & -391407.8 & 14.6 & 11.5 & $\mathrm{M} 1.75^{\mathrm{a}}$ & $3850^{\mathrm{a}}, 4400^{\mathrm{b}}$ & . \\
\hline SONYC-Lup3-3 & 160755.18 & -390604.0 & 13.5 & 11.4 & M 5.75, M6. $5^{\mathrm{c}}$ & $3050^{\mathrm{a}}, 2900^{\mathrm{b}}, 2950^{\mathrm{c}}$ & $\cdots$ \\
\hline SONYC-Lup3-4 & 160804.76 & -390449.6 & 13.9 & 12.4 & $\mathrm{M} 6.50^{\mathrm{a}}, \mathrm{M} 7^{\mathrm{c}}$ & $3000^{\mathrm{a}}, 2700^{\mathrm{b}}, 2900^{\mathrm{c}}$ & $\cdots$ \\
\hline SONYC-Lup3-5 & 160816.03 & -390304.5 & 12.5 & 11.1 & M $6.25^{\mathrm{a}}, \mathrm{M} 7.5^{\mathrm{d}}, \mathrm{M} 5.5^{\mathrm{g}}$ & $2950^{\mathrm{a}}, 2800^{\mathrm{b}}$ & Par-Lup3-1 \\
\hline SONYC-Lup3-7 & 160859.53 & -385627.8 & 13.9 & 12.7 & $\mathrm{M} 8.00^{\mathrm{a}, \mathrm{e}}, \mathrm{M} 8.5^{\mathrm{h}}$ & $2850^{\mathrm{a}}, 2600^{\mathrm{b}, \mathrm{h}}$ & $\cdots$ \\
\hline SONYC-Lup3-10 & 160913.43 & -385804.9 & 16.1 & 14.9 & $\mathrm{M} 8.75^{\mathrm{a}}$ & $2650^{\mathrm{a}}$ & $\cdots$ \\
\hline SONYC-Lup3-12 & 161118.47 & -390258.2 & 15.1 & 13.3 & $<\mathrm{M} 1^{\mathrm{a}}$ & $4200^{\mathrm{a}}$ & $\cdots$ \\
\hline EX Lup & 160305.5 & -401826 & 9.7 & 8.5 & $M 0^{f}$ & $\ldots$ & HD 325367, Th 14 \\
\hline $\mathrm{Sz}-88$ & 160700.61 & -390219.9 & 9.9 & 8.6 & $\mathrm{M} 1^{\mathrm{f}}, \mathrm{M} 0^{\mathrm{h}}$ & $3850^{\mathrm{h}}$ & Th 15 \\
\hline Sz-91 & 160711.57 & -390347.2 & 11.1 & 9.8 & $\mathrm{M} 0.5^{\mathrm{f}}, \mathrm{M} 1^{\mathrm{h}}$ & $3705^{\mathrm{h}}$ & Th 20 \\
\hline Sz-94 & 160749.68 & -390428.6 & 11.4 & 10.6 & $\mathrm{M} 4^{\mathrm{f}}, \mathrm{M} 4.5^{\mathrm{g}}$ & $\ldots$ & $\ldots$ \\
\hline Sz-95 & 160752.29 & -385805.6 & 11.0 & 10.0 & $\mathrm{M} 1.5^{\mathrm{f}}$ & $\ldots$ & $\ldots$ \\
\hline Sz-96 & 160812.48 & -390833.0 & 10.1 & 9.0 & $\mathrm{M} 1.5^{\mathrm{f}}, \mathrm{M} 2^{\mathrm{g}}$ & $3560^{\mathrm{g}}$ & $\ldots$ \\
\hline Sz-97 & 160821.84 & -390421.4 & 11.2 & 10.2 & $\mathrm{M} 3^{\mathrm{f}}, \mathrm{M} 4.5^{\mathrm{g}}, \mathrm{M} 4^{\mathrm{h}}$ & $3198^{\mathrm{g}}, 3270^{\mathrm{h}}$ & Th 24 \\
\hline Sz-98 & 160822.44 & -390446.7 & 9.5 & 8.0 & $M 0^{f}$ & $\ldots$ & $\ldots$ \\
\hline Sz-99 & 160824.00 & -390549.2 & 11.9 & 10.7 & $\mathrm{M} 3.5^{\mathrm{f}}, \mathrm{M} 4^{\mathrm{g}, \mathrm{h}}$ & $3270^{\mathrm{g}, \mathrm{h}}$ & Th 25 \\
\hline Sz-100 & 160825.76 & -390601.2 & 11.0 & 9.9 & $\mathrm{M} 5^{\mathrm{d}, \mathrm{f}}, \mathrm{M} 4.5^{\mathrm{g}}, \mathrm{M} 5.5^{\mathrm{h}}$ & $3198^{\mathrm{g}}, 3057^{\mathrm{h}}$ & Th 26 \\
\hline Sz-101 & 160828.37 & -390532.3 & 10.4 & 9.4 & $\mathrm{M} 4.5^{\mathrm{g}}, \mathrm{M} 4^{\mathrm{f}}$ & $3198^{\mathrm{g}}$ & Th 27 \\
\hline Sz-103 & 160830.24 & -390610.8 & 11.4 & 10.2 & $\mathrm{M} 4^{\mathrm{f}, \mathrm{h}}, \mathrm{M} 4.5^{\mathrm{g}}$ & $3198^{\mathrm{g}}, 3270^{\mathrm{h}}$ & Th 29 \\
\hline Sz-104 & 160830.72 & -390548.5 & 11.7 & 10.7 & $\mathrm{M}^{\mathrm{f}, \mathrm{h}}, \mathrm{M} 5.5^{\mathrm{g}}$ & $3058^{\mathrm{g}}, 3125^{\mathrm{h}}$ & Th 30 \\
\hline Sz-105 & 160837.03 & -401620.8 & 9.0 & 7.6 & $\mathrm{M} 4^{\mathrm{f}}$ & $\ldots$ & Th 31 \\
\hline Sz-106 & 160839.76 & -390625.3 & 11.7 & 10.1 & $\mathrm{M} 0^{\mathrm{f}}, \mathrm{M} 2.5^{\mathrm{d}}, \mathrm{M} 0.5^{\mathrm{h}}$ & $3777^{\mathrm{h}}$ & $\cdots$ \\
\hline Sz-107 & 160841.76 & -390136.8 & 11.2 & 10.3 & $\mathrm{M} .5^{\mathrm{f}}, \mathrm{M} 6.5^{\mathrm{g}}$ & $2935^{\mathrm{g}}$ & $\cdots$ \\
\hline Sz-108A & 160842.74 & -390618.4 & 9.8 & 8.8 & $\mathrm{M}^{\mathrm{d}}$ & $\ldots$ & $\cdots$ \\
\hline Sz-108B & 160842.87 & -390614.6 & $\ldots$ & $\cdots$ & M6 ${ }^{\mathrm{d}}, \mathrm{M} 5.5^{\mathrm{g}}$ & $3058^{\mathrm{g}}$ & $\cdots$ \\
\hline Sz-109 & 160848.16 & -390419.3 & 11.4 & 10.5 & $\mathrm{M} 5.5^{\mathrm{f}}, \mathrm{M} 6.5^{\mathrm{d}}$ & $\ldots$ & $\ldots$ \\
\hline Sz-110 & 160851.57 & -390317.7 & 11.0 & 9.7 & $\mathrm{M} 4.5^{\mathrm{d}}, \mathrm{M} 3^{\mathrm{g}}, \mathrm{M} 2^{\mathrm{f}}, \mathrm{M} 4^{\mathrm{h}}$ & $3415^{\mathrm{g}}, 3270^{\mathrm{h}}$ & Th 32 \\
\hline Sz-111 & 160854.74 & -393743.6 & 10.6 & 9.5 & $\mathrm{M} 1.5^{\mathrm{f}}, \mathrm{M} 1^{\mathrm{h}}$ & $3705^{\mathrm{h}}$ & Th 33 \\
\hline Sz-112 & 160855.53 & -390234.0 & 11.0 & 10.0 & $\mathrm{M} 4^{\mathrm{f}}, \mathrm{M} 6^{\mathrm{d}}, \mathrm{M} 5^{\mathrm{h}}$ & $3125^{\mathrm{h}}$ & \\
\hline Sz-113 & 160857.80 & -390222.8 & 12.5 & 11.3 & $\mathrm{M} 4^{\mathrm{f}}, \mathrm{M} 6^{\mathrm{d}}, \mathrm{M} 1.5^{\mathrm{g}}, \mathrm{M} 4.5^{\mathrm{h}}$ & $3633^{\mathrm{g}}, 3197^{\mathrm{h}}$ & Th 34, Lup 609s \\
\hline Sz-114 & 160901.85 & -390512.4 & 10.4 & 9.3 & $\mathrm{M} 5.5^{\mathrm{d}}, \mathrm{M} 4^{\mathrm{g}, \mathrm{f}}, \mathrm{M} 4.8^{\mathrm{h}}$ & $3270^{\mathrm{g}}, 3175^{\mathrm{h}}$ & Th 35 \\
\hline Sz-115 & 160906.23 & -390851.9 & 11.3 & 10.4 & $\mathrm{M} 4^{\mathrm{f}}, \mathrm{M} 4.5^{\mathrm{h}}$ & $3197^{\mathrm{h}}$ & $\ldots$ \\
\hline Sz-116 & 160942.61 & -391941.5 & 10.5 & 9.5 & $\mathrm{M} 1.5^{\mathrm{f}}$ & $\ldots$ & Th 36 \\
\hline Sz-117 & 160944.34 & -391330.4 & 10.7 & 9.4 & $\mathrm{M} 2^{\mathrm{f}}$ & $\ldots$ & Th 37 \\
\hline Sz-119 & 160957.07 & -385947.6 & 10.4 & 9.4 & $\mathrm{M} 4^{\mathrm{f}}$ & $\cdots$ & Th 38 \\
\hline Sz-121 & 161012.21 & -392118.6 & 10.0 & 9.0 & $M 3^{\mathrm{f}}$ & $\ldots$ & $\operatorname{Th} 40$ \\
\hline Sz-122 & 161016.44 & -390805.4 & 10.9 & 9.9 & $\mathrm{M} 2^{\mathrm{f}}$ & $\ldots$ & Th 41 \\
\hline Sz-123 & 161051.49 & -385314.1 & 11.1 & 9.8 & $M 1^{\mathrm{f}, \mathrm{h}}$ & $3705^{\mathrm{h}}$ & Th 42 \\
\hline Par-Lup3-2 & 160835.78 & -390347.9 & 11.2 & 10.3 & $\mathrm{M}^{\mathrm{d}}, \mathrm{M} 5^{\mathrm{g}}$ & $\ldots$ & $\cdots$ \\
\hline Par-Lup3-3 & 160849.40 & -390539.3 & 11.4 & 9.5 & $\mathrm{M} 4.5^{\mathrm{d}}, \mathrm{M} 4^{\mathrm{h}}$ & $3270^{\mathrm{h}}$ & $\ldots$ \\
\hline Par-Lup3-4 & 160851.44 & -390530.5 & 15.5 & 13.3 & $\mathrm{M} 5^{\mathrm{d}}, \mathrm{M} 4.5^{\mathrm{h}}$ & $3197^{\mathrm{h}}$ & $\ldots$ \\
\hline Lup $713 \mathrm{~s}$ & 160737.73 & -392138.8 & 13.2 & 12.1 & M5.75, M5. $5^{\mathrm{h}}$ & $3057^{\mathrm{h}}$ & $\cdots$ \\
\hline Lup 604s & 160800.17 & -390259.5 & 12.1 & 11.1 & $\mathrm{M} 5.25^{\mathrm{e}}, \mathrm{M} 5.5^{\mathrm{g}, \mathrm{h}}$ & $3058^{g, h}$ & $\ldots$ \\
\hline J16081497-3857145 & 160814.97 & -385714.5 & 15.2 & 13.1 & $\mathrm{M} 4.75^{\mathrm{e}}$ & $\ldots$ & $\ldots$ \\
\hline Lup 706 & 160837.33 & -392310.9 & 15.2 & 13.8 & $\mathrm{M} 7.75^{\mathrm{e}}, \mathrm{M} 7.5^{\mathrm{h}}$ & $2795^{\mathrm{h}}$ & $\ldots$ \\
\hline J16085373-3914367 & 160853.73 & -391436.7 & 15.0 & 12.5 & M5. $5^{\mathrm{e}}$ & $\cdots$ & $\cdots$ \\
\hline M-7 & 160855.20 & -384848.2 & 13.0 & 12.0 & M3 $^{\mathrm{g}}$ & $3415^{\mathrm{g}}$ & $\cdots$ \\
\hline M-17 & 160828.08 & -391309.8 & 13.7 & 12.4 & M5.5 & $3058^{\mathrm{g}}$ & Lup 607 \\
\hline M-18 & 160908.40 & -390342.8 & 12.2 & 11.4 & M5.5 g & $\ldots$ & Lup 608s \\
\hline M-19 & 160848.24 & -390919.4 & 12.9 & 12.0 & M6 $6^{\mathrm{g}}$ & $\ldots$ & Lup 617 \\
\hline M-20 & 160949.92 & -384902.6 & 15.4 & 14.4 & M3.5 g & $\ldots$ & Lup 650 \\
\hline M-23 & 160917.04 & -392709.7 & 13.5 & 12.7 & M4.5 g & $\ldots$ & Lup 710 \\
\hline M-24 & 160758.80 & -392434.9 & 12.6 & 11.6 & $\mathrm{M}^{\mathrm{g}}$ & $\ldots$ & Lup 714 \\
\hline M-25 & 161151.12 & -385105.0 & 13.3 & 12.3 & M5.5 & $\ldots$ & Lup 802 s \\
\hline M-27 & 160956.40 & -385951.0 & 13.0 & 12.0 & M6 $6^{\mathrm{g}, \mathrm{h}}$ & $2990^{\mathrm{g}, \mathrm{h}}$ & Lup 818 s \\
\hline M-51 & 161159.76 & -382338.4 & 12.2 & 11.2 & $\mathrm{M} 6^{\mathrm{g}}, \mathrm{M} 5^{\mathrm{h}}$ & $2990^{\mathrm{g}}, 3125^{\mathrm{h}}$ & SST-Lup3-1 \\
\hline M-58 & 160703.84 & -391111.4 & 14.7 & 13.1 & M5.5 & $3058^{\mathrm{g}}$ & $\ldots$ \\
\hline M-62 & 160901.44 & -392512.0 & 11.6 & 10.3 & $\mathrm{M} 3.5^{\mathrm{g}}, \mathrm{M} 4^{\mathrm{h}}$ & $3343^{\mathrm{g}}, 3270^{\mathrm{h}}$ & $\ldots$ \\
\hline M-65 & 161012.96 & -384616.3 & 15.9 & 13.9 & $\mathrm{M} 4^{\mathrm{g}}$ & $3270^{\mathrm{g}}$ & $\cdots$ \\
\hline M-66 & 161019.92 & -383606.5 & 13.3 & 12.3 & $M 6^{\mathrm{g}}$ & $2990^{\mathrm{g}}$ & $\ldots$ \\
\hline M-67 & 161029.52 & -392214.5 & 11.9 & 10.9 & M5. $5^{\mathrm{g}}$ & $3058^{\mathrm{g}}$ & $\ldots$ \\
\hline M-69 & 161131.92 & -381110.0 & 12.3 & 10.7 & M2.5 & $3488^{g}$ & $\cdots$ \\
\hline M-70 & 161144.88 & -383244.9 & 12.4 & 11.5 & M6. $5^{\mathrm{g}}$ & $2935^{\mathrm{g}}$ & $\ldots$ \\
\hline J16052862-3846210 & 160528.63 & -384621.1 & 12.6 & 11.8 & $M 6^{\mathrm{c}}$ & $3000^{c}$ & $\ldots$ \\
\hline J16083304-3852224 & 160833.05 & -385222.4 & 12.9 & 11.9 & $\mathrm{M} 7.5^{\mathrm{c}}$ & $2800^{c}$ & $\cdots$ \\
\hline
\end{tabular}




\begin{tabular}{|c|c|c|c|c|c|c|c|}
\hline & & & $\begin{array}{r}\text { Tabl } \\
\text { (Contin }\end{array}$ & & & & \\
\hline ID & $\begin{array}{c}\alpha \\
(\mathrm{J} 2000)\end{array}$ & $\begin{array}{c}\delta \\
(\mathrm{J} 2000)\end{array}$ & $\begin{array}{c}J \\
(\mathrm{mag})\end{array}$ & $\begin{array}{c}K \\
(\mathrm{mag})\end{array}$ & SpT & $\begin{array}{l}T_{\text {eff }} \\
(\mathrm{K})\end{array}$ & Other Names \\
\hline J16083547-3900358 & 160835.48 & -390035.8 & 11.5 & 10.6 & M6. $5^{\mathrm{c}}$ & $2950^{c}$ & $\ldots$ \\
\hline J16083974-3929228 & 160839.75 & -392922.9 & 11.7 & 10.9 & $\mathrm{M}^{\mathrm{c}}$ & $3100^{c}$ & $\cdots$ \\
\hline J16085575-3826330 & 160855.75 & -382633.1 & 13.0 & 12.1 & M5. $5^{\mathrm{c}}$ & $3050^{\mathrm{c}}$ & $\ldots$ \\
\hline J16090452-3921125 & 160904.52 & -392112.5 & 10.7 & 9.8 & $\mathrm{M}^{\mathrm{c}}$ & $3100^{\mathrm{c}}$ & $\ldots$ \\
\hline J16091570-3851396 & 160915.71 & -385139.7 & 12.0 & 11.2 & $\mathrm{M}^{\mathrm{c}}$ & $3100^{c}$ & $\cdots$ \\
\hline J16092279-3855506 & 160922.80 & -385550.6 & 10.8 & 9.9 & $\mathrm{M}^{\mathrm{c}}$ & $3100^{\mathrm{c}}$ & $\mathrm{J} 160922.8-385550 \mathrm{~A}^{\mathrm{c}}$ \\
\hline J16092320-3855547 & 160923.20 & -385554.8 & 10.8 & 9.9 & $\mathrm{M} 4^{\mathrm{c}}$ & $3250^{c}$ & $\mathrm{~J} 160922.8-385550 \mathrm{~B}^{\mathrm{c}}$ \\
\hline J16092800-3848538 & 160928.01 & -384853.8 & 11.9 & 11.0 & M5. $5^{\mathrm{c}}$ & $3050^{\mathrm{c}}$ & $\cdots$ \\
\hline J16101386-3759589 & 161013.87 & -375958.9 & 12.2 & 11.2 & $M 6^{\mathrm{c}}$ & $3000^{c}$ & $\ldots$ \\
\hline J16103062-3831517 & 161030.62 & -383151.7 & 13.3 & 12.4 & $M 6^{\mathrm{c}}$ & $3000^{c}$ & $\ldots$ \\
\hline J16103323-3830234 & 161033.23 & -383023.5 & 10.8 & 9.8 & $\mathrm{M} 4.5^{\mathrm{c}}$ & $3200^{c}$ & $\cdots$ \\
\hline J16104192-3823046 & 161041.92 & -382304.7 & 10.9 & 10.0 & $\mathrm{M}^{\mathrm{c}}$ & $3100^{\mathrm{c}}$ & $\cdots$ \\
\hline J16105899-3914514 & 161058.99 & -391451.4 & 12.1 & 11.3 & $\mathrm{M}^{\mathrm{c}}$ & $3100^{c}$ & $\ldots$ \\
\hline J16113801-3841356 & 161138.02 & -384135.7 & 10.9 & 10.1 & $\mathrm{M} 2.5^{\mathrm{c}}$ & $3500^{c}$ & $\cdots$ \\
\hline J16120761-3813242 & 161207.61 & -381324.3 & 10.9 & 10.0 & $\mathrm{M} 4.5^{\mathrm{c}}$ & $3200^{c}$ & $\ldots$ \\
\hline J16121046-3909040 & 161210.46 & -390904.0 & 13.9 & 12.8 & M6. $5^{\mathrm{c}}$ & $2950^{c}$ & $\ldots$ \\
\hline J16122559-3817428 & 161225.58 & -381742.8 & 13.1 & 12.2 & $\mathrm{M}^{\mathrm{c}}$ & $3000^{c}$ & $\ldots$ \\
\hline
\end{tabular}

Notes. Identifiers are from Schwartz (1977, Sz), Comerón et al. (2003, Par-Lup3), López Martí et al. (2005, Lup), Mortier et al. (2011, M), and 2MASS (J16).

References. (a) this work, (b) Comerón et al. 2009; (c) Comerón et al. 2013; (d) Comerón et al. 2003; (e) Allen et al. 2007; (f) Hughes et al. 1994; (g) Mortier et al. 2011; (h) Alcalá et al. 2014.
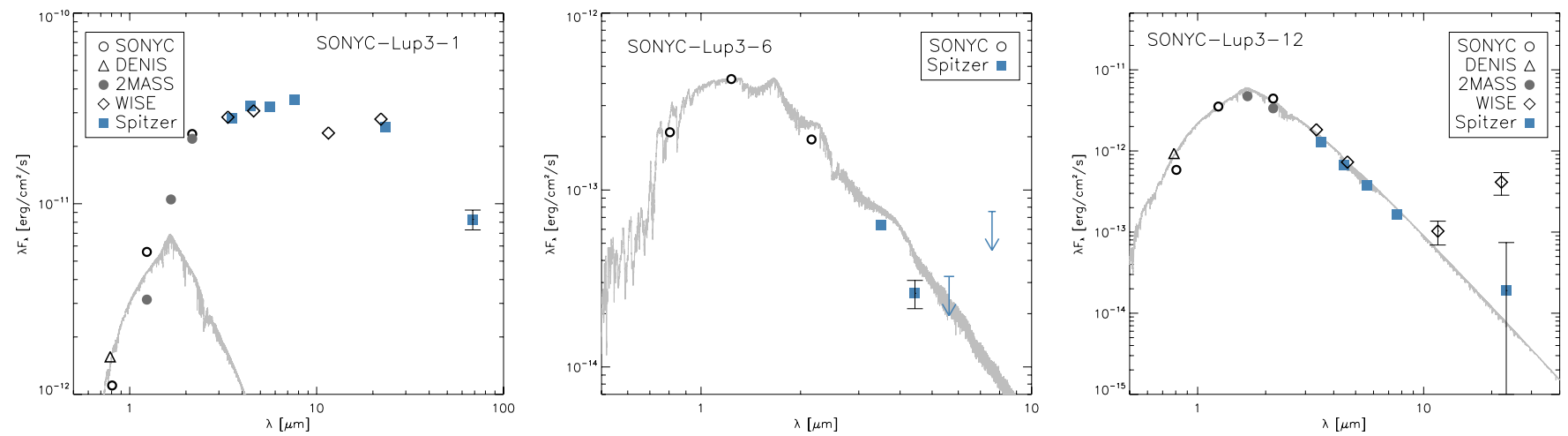

Figure 11. Spectral energy distribution of SONYC-Lup3-1, -6, and -12. Photometry comes from this work (open circles), DENIS (triangles), 2MASS (filled gray circles), Spitzer IRAC and MIPS (squares), and WISE (diamonds). The uncertainties are comparable to or smaller than the size of the symbols, except for the points with the error bars shown.

(A color version of this figure is available in the online journal.)

report the underluminosity of Par-Lup3-4, and a jet associated with Par-Lup3-4 was discovered in [S II] images by Fernández \& Comerón (2005). Comerón et al. (2003) discuss three possible interpretations for the underluminosity and emission line spectra of the two objects:

(1) an edge-on disk blocking a part of the stellar light, (2) embedded Class I sources where the star is seen via the light scattered by the walls of cavities in the envelope, and (3) accretion-modified evolution, similar to what is described in Hartmann (1998) and Baraffe et al. (2009). High-resolution spectra of Par-Lup3-4 (Fernández \& Comerón 2005) reveal a double-peaked [S II] profile, implying that the low excitation jet is seen at a small angle with respect to the plane of the sky. The authors argue that at this inclination, only a flared disk could hide a star. Huélamo et al. (2010) studied the target spectral energy distribution (SED) from the optical to the submillimeter regime and compared it with a grid of radiative transfer models of circumstellar disks. They find that Par-Lup34 is a Class II source, with an edge-on disk that naturally explains its underluminosity.
In Figure 11 we show the SED of SONYC-Lup3-1 in the range $0.8-70 \mu \mathrm{m} .{ }^{14}$ Open circles mark the optical and NIR photometry from the SONYC campaign, the filled grey circles show the 2MASS NIR photometry, squares denote the Spitzer MIR data, and diamonds denote the MIR photometry from WISE. The double-peaked shape of the SED with a dip around $10 \mu \mathrm{m}$ is characteristic for an edge-on disk, where the NIR peak arises from the scattered light of the star obscured by a disk, and the emission at longer wavelengths comes from the dust (e.g., Wood et al. 2002; Pontoppidan et al. 2007; Huélamo et al. 2010). The radiative transfer SED modeling could shed more light on the properties of the putative disk around SONYCLup3-1, but since this is out of the scope of this paper, we refrain from discussing this source further. To calculate the luminosity of SONYC-Lup3-1 in Figure 10, we used the $A_{V}=3.0 \mathrm{mag}$ derived from fitting a model to the spectrum. The extinction calculated from the $J-K$ color is, however, significantly higher $\left(A_{V}=11.5 \mathrm{mag}\right)$ and shifts the point in the H-R diagram in the

\footnotetext{
$\overline{14}$ Constructed with the help of VOSA (Bayo et al. 2008).
} 
$y$-direction such that it falls roughly onto the $10 \mathrm{Myr}$ isochrone. We conclude that SONYC-Lup3-1 is probably a member of Lupus 3, with properties similar to Par-Lup3-4 and Sz 102. The MIR-excess and the double-peaked SED suggest a presence of an edge-on circumstellar disk.

\subsection{SONYC-Lup3-6}

We obtained the spectrum of SONYC-Lup3-6 by chance alignment of the slit that was placed on SONYC-Lup3-7, which belongs to our high-priority candidate list. SONYC-Lup3-6 is located $\sim 7^{\prime \prime}$ north of SONYC-Lup3-7. With the $i-J$ color of 1.8, SONYC-Lup3-6 is too blue to be in our photometric candidate list, i.e., the colors suggest that the object is not a pre-main-sequence source. In the H-R diagram, it falls well below the $100 \mathrm{Myr}$ isochrone. However, the spectral fitting procedure identifies the object as a young star of the spectral type M4.75. It shows low-gravity $\mathrm{Na}$ I signatures, $\mathrm{H}_{\alpha}$ emission, and no evidence for Ca II triplet absorption. Furthermore, its proper motion satisfies the proper motion membership criterion. From the $\mathrm{EW}\left(\mathrm{H}_{\alpha}\right)$ it is not clear whether SONYC-Lup3-6 undergoes accretion or not, as it falls close to the line that separates accretors from non-accretors (yes according to the criterion by Barrado y Navascués \& Martín 2003, and no according to White \& Basri 2003). If it is indeed a member of Lupus 3, SONYC-Lup3-6 might be a primary of a very wide binary system containing the M8 BD SONYC-Lup3-7. The projected separation of this hypothetical system would be $\sim 1400 \mathrm{AU}$. It would be, however, very difficult to explain a M4.75 primary that is several magnitudes fainter than its supposed secondary. We therefore classify the membership of SONYC-Lup3-6 as uncertain.

\subsection{SONYC-Lup3-12}

This object's spectrum suggests a type earlier than M1 but was nevertheless selected in our preliminary analysis on the basis of the obvious $\mathrm{H}_{\alpha}$ emission observed in its spectrum (Figure 8). According to West et al. (2008), early M-dwarfs have shorter activity lifetimes than the later M-dwarfs, which speaks in favor of SONYC-Lup3-12 being indeed young. However, as in the case of SONYC-Lup3-6, the $\operatorname{EW}\left(\mathrm{H}_{\alpha}\right)$ falls right at the border separating accreting objects from non-accretors (accretor according to the criterion by Barrado y Navascués \& Martín 2003, and non-accretor according to White \& Basri 2003). When compared with the spectra of other objects in our sample with similar early spectral type (e.g., SONYC-Lup3-17, -22, -23), we notice the lack of the Ca II absorption lines, i.e., this object is likely not to be a giant. On the basis of this evidence, we tentatively include SONYC-Lup3-12 in the final list of the confirmed members. It shows no MIR-excess, and therefore the edge-on disk morphology does not seem to be a plausible explanation for its underluminosity.

\subsection{Summary of the Results}

From the candidate list containing 409 objects in the direction of Lupus 3 cloud, 123 objects were selected for the spectroscopic follow-up; 27 showed spectral features consistent with spectral type $\mathrm{M}$ or slightly earlier. Fitting the spectra to an empirical grid of spectra of young dwarfs, field dwarfs, and giants, combined with the proper motion assessment of membership, and the positions of the candidates in the HR diagram, we identify nine field dwarfs, four background giants, and seven probable members of Lupus 3, among which two show significant

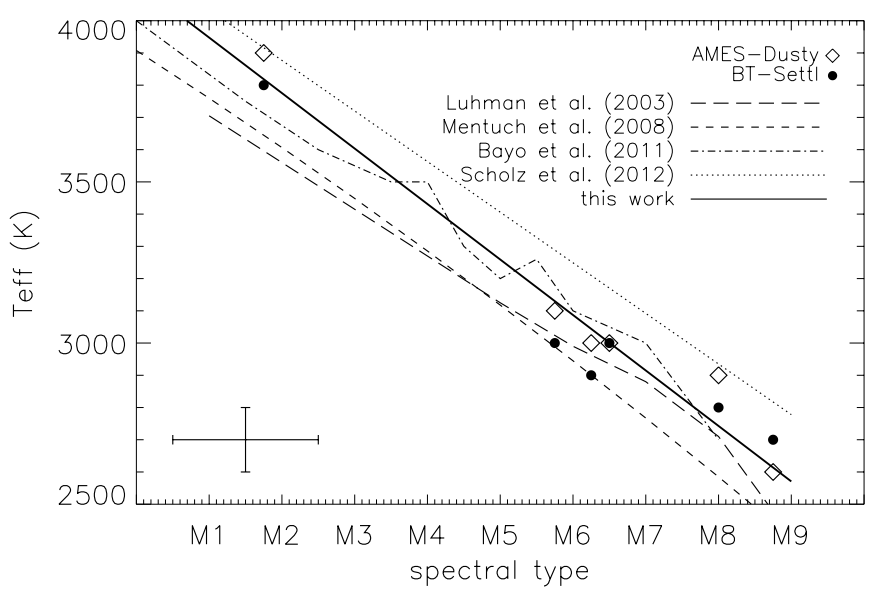

Figure 12. Comparison between the spectral types and effective temperatures for the probable members of Lupus 3 with $T_{\text {eff }}<4000 \mathrm{~K}$ confirmed in this work. Two sets of symbols stand for effective temperature that were determined by fitting different models to our spectra (see Table 3 ). The typical error bar is shown in the lower left corner of the plot. The solid line is a linear fit to the data points. We also show the effective temperature scales by Luhman et al. (2003b), Mentuch et al. (2008), Bayo et al. (2011), and Scholz et al. (2012b).

underluminosity for their spectral type. The nature of seven sources remains uncertain. In Table 2 we list the 27 analyzed sources and give their coordinates, photometry, spectral types, proper motions, $\mathrm{EW}\left(\mathrm{H}_{\alpha}\right)$, membership status, and references for those identified in the literature.

\section{RESULTS AND DISCUSSION}

In this section we use the members of Lupus 3 confirmed in this work to derive a relation between spectral type and $T_{\text {eff }}$, and we combine the outcome of our survey with the results from other works, in order to establish a census of VLM objects in Lupus 3, and discuss the properties of the substellar population.

\subsection{Effective Temperature vs. Spectral Type}

In Figure 12, we plot the relation between $T_{\text {eff }}$ and the spectral type for the objects classified as members of Lupus 3 and with $T_{\text {eff }}<4000 \mathrm{~K}$ (SONYC-Lup3-1, -3, -4, -5, -7, and -10). The upper limit in $T_{\text {eff }}$ is set because our spectral type fitting scheme does not extend to spectral types earlier than M1. Each object listed as member in Table 2 is represented by two data points, one for the $T_{\text {eff }}$ derived using BT-Settl models (filled circles) and the other using AMES-Dusty (open diamonds). The solid line represents a linear fit to all the data points:

$$
T_{\text {eff }}=(4120 \pm 175)-(172 \pm 26) \times \mathrm{SpT},
$$

where SpT corresponds to the M subtype. A linear least-square fit is performed using the fitexy procedure from Press et al. (2002), which takes into account both the uncertainties in spectral type and $T_{\text {eff }}$, shown in the lower left corner of Figure 12. The reduced $\chi^{2}$ of the fit is 2 , with the $q$ parameter of 0.996 . $^{15}$ We also show the effective temperature scales available from other works. The long-dashed line shows the scale from Luhman et al. (2003b) derived for IC348, while the dashed line represents the scale derived for the nearby young stellar associations by Mentuch et al. (2008). The latter scale is extrapolated below

\footnotetext{
15 The $q$-parameter stands for the probability that a correct model would give a value equal or larger than the observed $\chi^{2}$. It is a scalar with values between 0 and 1 , where a small value of $q$ indicates a poor fit.
} 
$3000 \mathrm{~K}$. The dash-dotted line shows the scale derived from the spectroscopic data of Collinder 69 (Bayo et al. 2011), and the dotted line is a scale for NGC 1333, derived by using the $H$-band peak index (HPI) defined in Scholz et al. (2012b). This scale was extrapolated to the spectral types earlier than M6, since the HPI index is defined only for later spectral types. We note that the scale of Scholz et al. (2012b) was derived from the near-infrared data, while all the rest are based on optical spectroscopy.

Although the trend seen in our data is generally consistent with all the previous works, some systematic offsets between the classification schemes is evident. The HPI index seems to give systematically later spectral types for a given $T_{\text {eff }}$ than other schemes. The same trend was already reported in the Mužić et al. (2012), where we used the HPI to derive spectral types from NIR spectra in highly extinct cluster $\rho$ Oph. There we speculated that the extinction might introduce additional uncertainties to spectral type derived from the HPI, because the index was defined on a sample of VLMOs in NGC 1333, a cluster with $A_{V}$ typically below 5. Lupus 3, however, has extinction levels comparable to those of NGC 1333, while we observe the same trend toward later spectral types. It seems therefore that for HPI the trend persists irrespective of differences in extinction of the samples and wavelength ranges used for spectral classification (optical versus NIR). A widely used relation between spectral type and $T_{\text {eff }}$ from Luhman et al. (2003b) seems to deliver systematically lower $T_{\text {eff }}$ for the same spectral type, compared with the relation derived here, especially for spectral types earlier than M6.

\subsection{Census of Very Low Mass Members of Lupus 3}

The most recent summary of the population of the Lupus star-forming region appeared in Comerón (2008). Since then, however, various studies have identified new low-mass members, and most importantly, there have been a significant efforts to provide spectroscopic confirmation of photometrically identified candidates. We therefore think it is timely to create a summary of the previous studies, together with results of our work, and provide a census of the VLM content of Lupus 3. In Table 4, we list coordinates, photometry, spectral type and/or $T_{\text {eff }}$, and alternative names for all spectroscopically confirmed members with the spectral type M0 or later. Since we choose to concentrate only on the spectroscopically confirmed members, we do not include the photometric candidates from the studies such as Nakajima et al. (2000) and López Martí et al. (2005), although we do include the identifiers from the latter study, in case they were spectroscopically confirmed in other works.

The starting point for this section is the review paper on the content of the Lupus clouds by Comerón (2008). Table 11 of the review contains a list of all classical T-Tauri stars in Lupus 3 known to the date of publication. This list includes all the bright members identified and confirmed in studies by The (1962), Schwartz (1977), Krautter (1992), Hughes et al. (1994), and Krautter et al. (1997), among which are 33 M-type stars.

Comerón et al. (2003) surveyed a small area $\left(\sim 10^{\prime} \times 5^{\prime}\right)$ surrounding the two brightest members HR5999 and HR6000, using slitless spectroscopy followed by the MOS follow-up using FORS/VLT. They find four M-type members, including Par-Lup3-1 (SONYC-Lup3-5), which the authors label a bonafide BD because of the assigned spectral type of M7.5 and the presence of the signatures of youth. Our spectral classification assigns it a slightly earlier spectral type (M6.25). The difference in the spectral types might arise from the different spectral typing schemes, especially since the typing in Comerón et al. (2003) was done by comparison with the field stars from Kirkpatrick et al. (1991), as not many young, low-gravity BDs were known at the time. The $T_{\text {eff }}$ derived from the model fitting is, on the other hand, in excellent agreement with $2800 \pm 200 \mathrm{~K}$ derived from the SED modeling in the optical and near-infrared in Comerón et al. (2009).

Allen et al. (2007) spectroscopically confirmed six candidate members selected from Spitzer MIR photometry, one of which was also in our high-priority selection box (SONYC-Lup3-7). Allen et al. (2007) classify SONYC-Lup3-7 as M8, in agreement with the spectral type obtained in this work.

Merín et al. (2008) selected 124 candidate members in Lupus 3 from the $c 2 d$ Spitzer IRAC observations. Part of this sample (46 objects) was spectroscopically followed-up with FLAMES/VLT by Mortier et al. (2011). From the 46 objects, 8 are discarded as non-members because they lie far above the premain-sequence evolutionary tracks. For the three objects found below the tracks, the authors propose an envelope or an edgeon disk geometry in case of membership in Lupus. Otherwise, these would be sources located in the background. One of these three objects is a well-known underluminous member of Lupus 3 (Sz 102) and an emitter of forbidden lines (see Section 4.7 for more details). We choose to include one of the two remaining objects to the compiled list of members (M-65), since it exhibits MIR-excess, $\mathrm{H}_{\alpha}$ emission, and spectral type later than M0. The third object is classified as K2 (M-71) and thus not included in the census table.

Two of the spectroscopically confirmed objects from Mortier et al. (2011) are found within our photometric selection box, and for one of them we also obtained a spectrum. This is SONYCLup3-5 (Par-Lup3-1), which Mortier et al. classify as M5.5. In this work the same object is classified as M6.25 and by Comerón et al. (2003) as M7.5. Spectra used in Mortier et al. (2011) span only the blue part of our spectral range (6550-7150 $\AA$ ), which might be the cause of the different spectral classification, as the molecular features at $\lambda>7200 \AA$ show more dramatic change with spectral type than the blue portion of the optical spectra.

Comerón et al. (2009) identified 72 candidate members of Lupus 3 with $T_{\text {eff }}<3400 \mathrm{~K}$. Recently, a spectroscopic followup of 46 out of 72 candidate members by Comerón et al. (2013) revealed that about $50 \%$ of the objects are background giants. Only four objects from the sample of Comerón et al. (2009) are in the SONYC area and are not affected by saturation and data reduction artifacts in our optical images. One of these four objects (16:09:20.8, -38:45:10) appears elongated in both MOSAIC and NEWFIRM images and thus did not end up in our final $i J$ catalog. Its spectrum by Comerón et al. (2013) shows that it is probably an M-type field dwarf. The remaining three objects are all found in our high-priority candidate list (photometric and proper motion selection), two of which were observed spectroscopically and indeed confirmed as members (SONYC-Lup3-3 and -4).

Recently, Alcalá et al. (2014) published the VLT/X-shooter spectroscopy of several Class II YSOs in Lupus 3, selected from the lists of Merín et al. (2008) and Allen et al. (2007). Because of the exceptionally wide wavelength range of X-shooter spectra (UV to NIR), and their resolution, we expect that the spectral types derived from these observations should be highly reliable, and they are included in the census in Table 4.

\subsection{Substellar Population in Lupus 3}

On the basis of the survey presented in this work, we can put limits on the number of VLM sources that are missing in the 

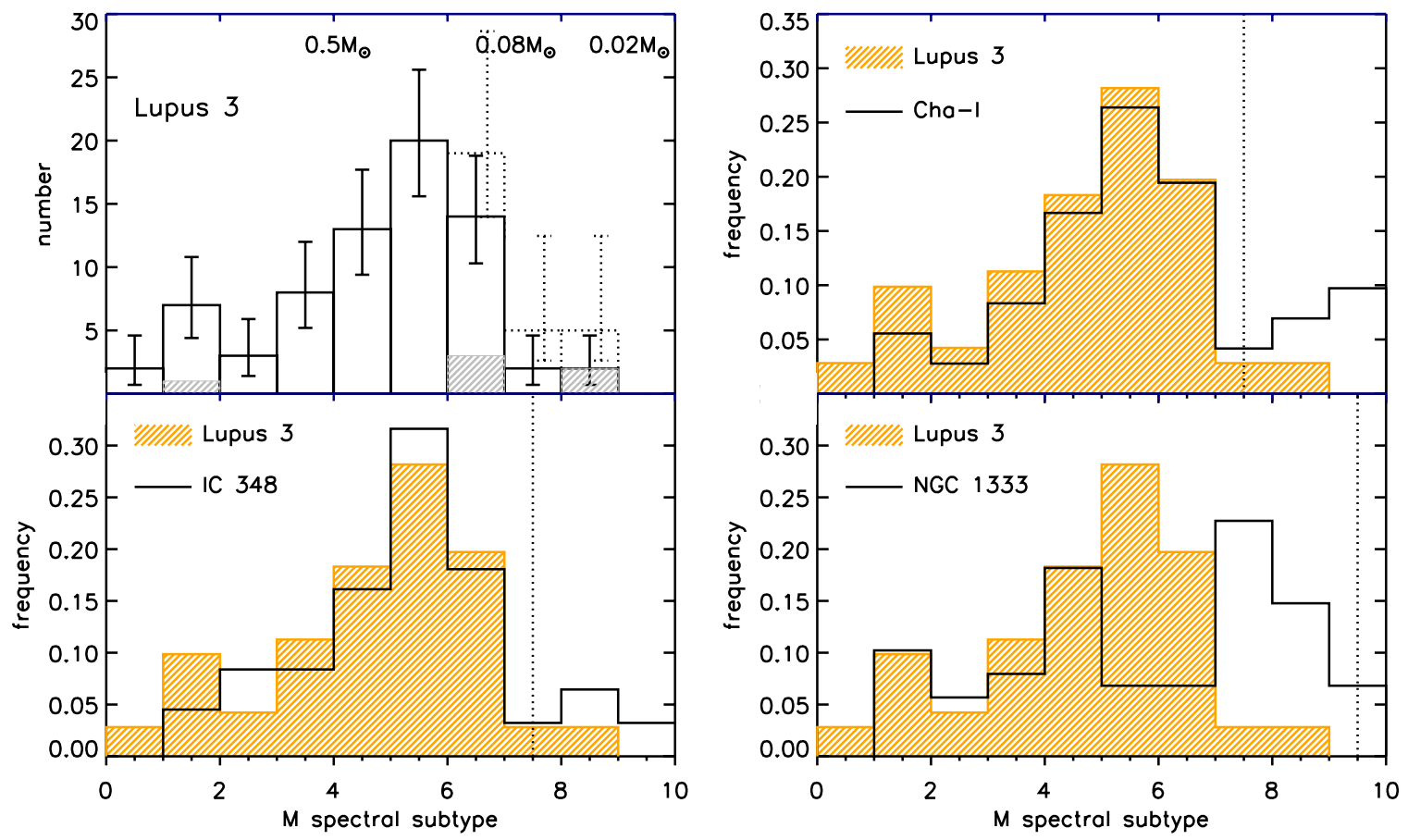

Figure 13. Top left: distribution of spectral types for the VLM population of Lupus 3, featuring the M-type sources from Table 4 within the field covered by our survey. The shaded histogram shows the SONYC contribution to the census. Dotted bins take into account the estimate of the objects probably missing in our survey (for clarity, the associated error bars are displaced to the right of the uncertainty in the number of confirmed sources). The rough mass limits according to BT-Settl and AMES-Dusty isochrones are shown on top of the plot. Top right and two bottom panels: comparison between the spectral type distribution in Lupus 3 and those in Cha-I, IC 348 (Luhman 2007) and NGC 1333 (Scholz et al. 2012b).

(A color version of this figure is available in the online journal.)

current census of YSOs in Lupus 3. To avoid a possible bias toward the objects with disks, we base the following discussion only on the $I J$ candidate list and take into account only the objects above the completeness limit of our survey (dotted line in Figure 2). The completeness limit of $i=20.3$ is equivalent to $0.009-0.02 M_{\odot}$ for $A v=0-5$, at a distance of $200 \mathrm{pc}$ and age of 1 Myr, according to the BT-Settl models. The limits translate to $0.007-0.02 M_{\odot}$ in the case of AMES-Dusty and $0.005-0.015 M_{\odot}$ in case of AMES-COND models. ${ }^{16}$

In the candidate selection box in Figure 2, we find 342 candidates above the completeness limit. Of these, 54 are also proper motion candidates (i.e., belong to the "IJ-pm" list). We obtained 111 spectra for the photometrically selected candidates, from which 31 are also proper motion candidates. The seven objects confirmed to be Lupus 3 members in this work are all from the proper motion selection. To this we add three previously confirmed members found inside the box (green stars in Figure 2), from which one is a proper motion candidate.

Our experiment is an example of a random variable, following the binomial probability distribution. On the basis of the observed number of success counts in our sample, we can determine the maximum-likelihood value for the frequency of substellar objects in the sample, which is basically the ratio between the confirmed objects and those objects with spectra. The 95\% confidence intervals (CI) are calculated using the Clopper-Pearson method, which is suitable for smallnumber events and returns conservative CIs compared with other methods (Gehrels 1986; Brown et al. 2001) The success rate in

16 Note that the version of DUSTY and COND models with updated opacities, BT-Dusty and BT-COND, do not extend below $0.03 M_{\odot}$ at early ages. the "IJ-pm" sample is therefore $8 / 32=25_{-14}^{+18} \%$, while for the photometric candidates it is $2 / 82 \approx 2_{-2}^{+6} \%$. In total, the estimated number of unconfirmed members in our survey is $(54-32) \times 0.25+(342-54-82) \times 0.02=6+5=11$, with the $95 \% \mathrm{CI}$ of ${ }_{-5}^{+13}$. We can repeat the same calculation to get an approximate number of missing BD above the completeness limit. From the SONYC sources, three are most probably BDs (all existing spectral classifications $\geqslant M 6$ ), while two have spectral types around the substellar border. Among the three sources from other works, one is a star earlier than M0, one is classified as M7.5 (J16083304-3855224 in Table 4), and the remaining one is classified as M6 (M-66). The total number of spectroscopically confirmed BDs in our sample of 342 low-mass candidates above the completeness limit is therefore between 4 and 7, and only M-66 does not come from the proper motion selection. The calculation for missing BDs is identical to the one above but with different success rates. For the "IJ-pm" sample we have 4 to 6 BDs over 32 objects and for the photometric candidates 0 to $1 \mathrm{BD}$ over 82 . The estimated number of unconfirmed BDs is then $6_{-3}^{+10}$.

\subsection{Spectral Type Distribution}

In Figure 13 we show the distribution of spectral types for the spectroscopically confirmed M-type population in Lupus 3, found within the field covered by our survey (top left panel; solid lines). The solid error bars are Poissonian CIs calculated with the method described in Gehrels (1986), and the dashed histogram shows the contribution of our survey to the census. The dotted histogram includes the estimate on the number of missing objects estimated in Section 5.3, assuming that 6 of 
the objects are BDs (i.e., found somewhere in the last two bins and equally distributed for the purpose of this plot) and the remaining five are probably low-mass stars, which because the saturation limit of our survey is around $0.06 M_{\odot}$, should fall in the bin above. The dotted-line error bars combine in quadrature the CI from the binomial distribution, with the error bars shown with solid lines.

We also show a comparison with the results from other star-forming regions: Cha-I (Luhman 2007; top right), IC 348 (Luhman 2007; bottom left), and NGC 1333 (Scholz et al. 2012a; lower right). In the stellar regime, the distribution resembles those seen in other star-forming regions, with the peak around M5-M6 and an increase in the number of objects between M0-M1 and M5 by a factor of 2-3. Cha-I and IC 348 show a drop in the number of objects in the substellar regime with respect to stars, and this is at a similar level seen also in Lupus 3. Although this sharp drop at spectral types M7 and later can partially be explained by the incompleteness of our survey at lowest masses, it is likely to be a real feature of the IMF considering the analysis of the missing objects in our survey presented in the previous section.

In NGC 1333 (Scholz et al. 2012a), we see a second peak around $\mathrm{M} 7$, which might be due to a gap in the completeness between two different spectroscopic samples that were used in the stellar and substellar regimes, causing us to miss the objects around M6. The double peak is also seen in the distribution of absolute $J$-magnitudes, but this distribution is consistent, within statistical errors, with a single broad peak. On the substellar side, the SONYC survey in NGC 1333 is deeper than the surveys in Cha-I, IC 348, and Lupus 3, and the completeness limit is in fact outside the $x$-axis range in Figure 13. From the statistical analysis in the previous section, given the relatively large errors, we can say that the SpT distribution down to M9 is consistent with that of NGC 1333.

\subsection{Star/BD Ratio}

In this section we use the results of our survey, combined with previous works to get some constraints on the IMF in Lupus 3, extending down to the completeness limit of our $i J$ catalog. In the following, we will take into account the area of our combined $i J$ survey (solid line in Figure 2). We note that the number of missing objects calculated in the previous section is partially based on the success rates of the candidates selected from the proper motions, i.e., from a somewhat smaller area than that of the photometric survey. We, however, choose not to apply any correction to account for this because, from Figure 2, it is evident that the majority of the high-priority candidates concentrate in the cloud core, and very few candidate members are expected to be missed in the surroundings. The surveys of Merín et al. (2008) and Comerón et al. (2009) have a comparable sky coverage in the area around the main Lupus 3 core, and they also cover the northeastern clouds of lower density, which are also considered to be part of the complex. This portion of the cloud contains a lower concentration of members (only about $10 \%$ of all the members identified in Merín et al. 2008 and Comerón et al. 2009), and thus excluding it should not significantly affect our analysis.

For this calculation, we compile a list of all members and candidate members from the two most extensive surveys found in the literature, Merín et al. (2008) and Comerón et al. (2009), and combine it with the sources identified in this work. As for the other works, we note that almost all sources from Table 11 of Comerón (2008) and Comerón et al. (2003), and all the confirmed sources by Allen et al. (2007), are already included either in the Merin et al. list or the SONYC list. We also include the estimated number of still undiscovered stellar and substellar members of Lupus 3, as determined in the previous section.

Mortier et al. (2011) confirm $\sim 83 \%$ of the MIR-excess sources from Merín et al. (2008) as probable members of Lupus 3, while the confirmation rate for the Comerón et al. (2009) sample is $\sim 50 \%$ (Comerón et al. 2013). These factors are taken into account when estimating the total number of probable members of the two mentioned studies. Another important correction factor that has to be taken into account is the disk fraction, which according to Merín et al. (2008) is 70\%-80\% for Lupus. Therefore, the number of objects from this work is additionally multiplied by a factor of 1.25 . The sample from Merín et al. (2008) is complete down to $0.1 M_{\odot}$ and nicely complements the one of Comerón et al. (2009), whose method is only sensitive to the objects cooler than $3400 \mathrm{~K}$. The $3 \sigma$ detection limit of Comerón et al. (2009) is at $I_{C}=22.2$, but the completeness limits are not specified. However, given the depth of their observations, it is probably safe to assume that they are complete in the range of masses between $0.1 M_{\odot}$ of Merín et al. (2008), and the upper limit in mass set by saturation in our optical data $\left(\sim 0.06 M_{\odot}\right)$.

To assess the numbers of stars and BDs, we use the approach described in Scholz et al. (2013). In short, by comparing the multi-band photometry with the predictions of the evolutionary models (BT-Settl in this case), we derive best-fit mass and $A_{V}$ for each object, for the assumed distance of $200 \mathrm{pc}$, age of $1 \mathrm{Myr}$, and the extinction law from Cardelli et al. (1989) with $R_{V}=4$. We note that the several underluminous objects identified here and in previous studies are erroneously identified by this procedure as BDs. However, these are clearly stars and therefore are counted in this higher-mass bin.

All the objects with estimated masses below $0.065 M_{\odot}$ are counted as BDs and all those above $0.085 M_{\odot}$ as stars. The remaining objects at the border of the substellar regime are once included in the higher-mass bin and then in the lower-mass bin. The calculated number of stars is then 80-92, and the number of BDs is estimated to be 28-40. Finally, we obtain the star/BD ratio between 2.0 and 3.3, in line with other star-forming regions, which typically show a span of values between 2 and 6 (Scholz et al., 2013, in press). Clearly, there are a number of uncertainties involved in this calculation because of possible incompleteness at the overlap of the different studies and uncertainties in age and distance, as well as the choice of the isochrones used to derive masses. It is nevertheless a useful exercise at least for a first order comparison with other works.

\section{SUMMARY AND CONCLUSIONS}

In this work, we have presented deep optical and near-infrared images of the $1.4 \mathrm{deg}^{2}$ area surrounding the two brightest members HR5999/6000 of the Lupus 3 star-forming region. From the optical+NIR photometry we selected 409 candidate VLM cluster members. Proper motion analysis, based on two epochs of imaging separated by 11-12 years, helped to narrow down the candidate selection to 59 high-priority candidates. To confirm the membership of the selected candidates, we performed spectroscopic follow-up using VIMOS/VLT, in which we collected 123 spectra from the photometric selection box, including 32 from the high-priority list. We confirm seven candidates as probable members of Lupus 3, among which four are later than M6.0 and with $T_{\text {eff }} \leqslant 3000 \mathrm{~K}$, i.e., are probably substellar in 


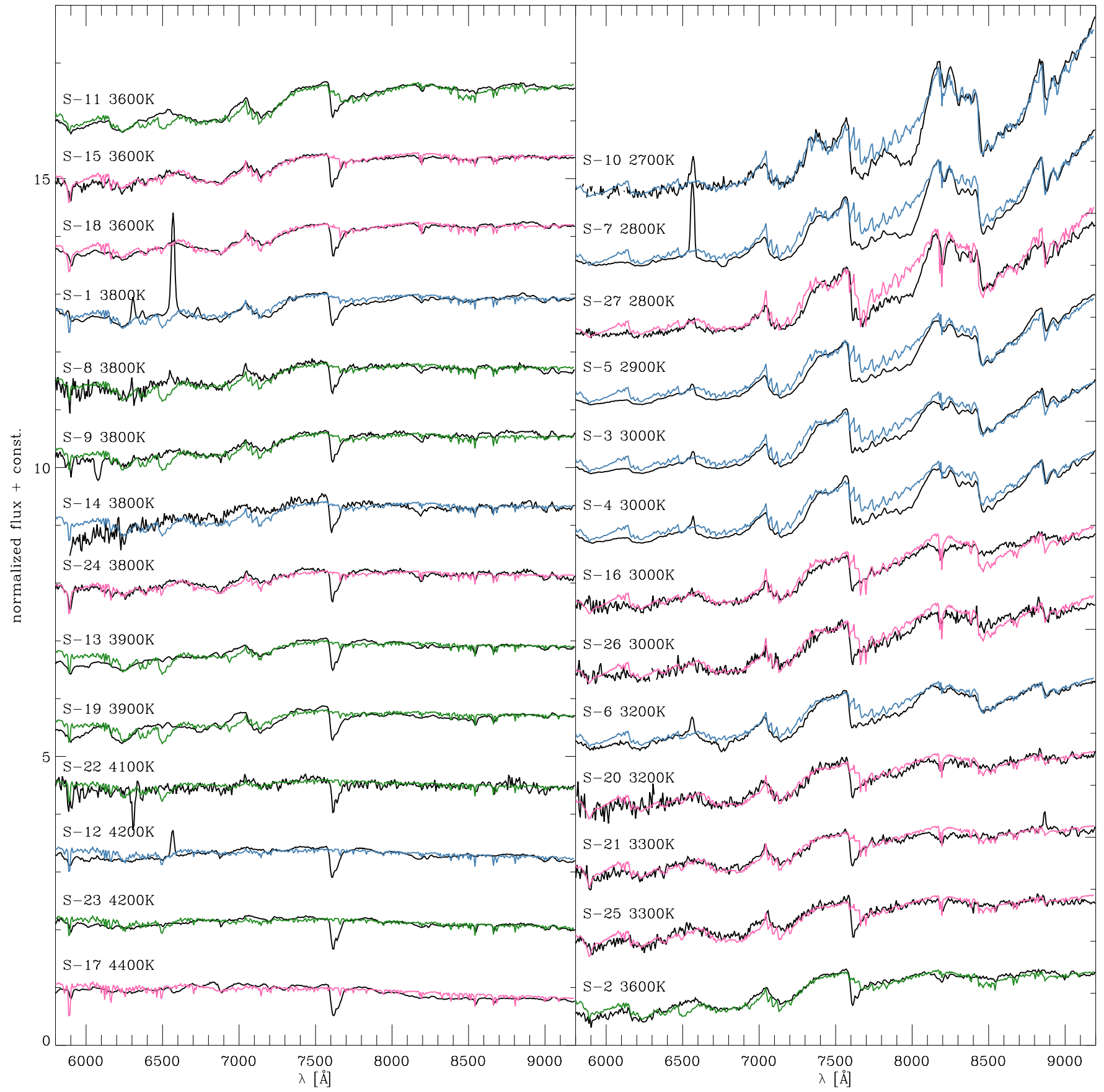

Figure 14. Spectra of the objects in Table 2 (black) and the best-fit BT-Settl model (Allard et al. 2011). The model spectra are color-coded according to the log $(g$ ) used in fitting: green assume $\log (g)=3.0$, blue assume $\log (g)=3.5$, and pink assume $\log (g)=5.0$. The spectral resolution of the models has been reduced by boxcar smoothing in order to match the resolution of our spectra. Each spectrum has been corrected for extinction but not for the atmospheric absorption.

(A color version of this figure is available in the online journal.)

nature. Two of the sources identified as probable members of Lupus 3 appear underluminous for their spectral class, similar to some previously known members exhibiting similar emission line spectra with strong $\mathrm{H}_{\alpha}$ and several forbidden lines associated with active accretion.

We derive a relation between the spectral types (from comparison to low-gravity objects in young regions) and $T_{\text {eff }}$ (from BT-Settl models): $T_{\text {eff }}=4083-166 \times \mathrm{SpT}$, where SpT refers to the $\mathrm{M}$ spectral subtype between 1 and 9 . The rms-error of the fit is $61 \mathrm{~K}$. We derive a star-to-BD ratio of 2.0-3.3, consistent with the values observed in other star-forming regions.
Combining our results with previous work on Lupus 3, we compile a table containing all spectroscopically confirmed lowmass objects with spectral type M0 or later, and we show that the distribution of spectral types is in line with what is observed in other young star-forming regions.

We thank Kevin Luhman and Amelia Bayo for sharing the spectra of young low-mass objects. This work was co-funded under the Marie Curie Actions of the European Commission (FP7KCOFUND). A.S. acknowledges financial support through the grant 10/RFP/AST2780 from the Science Foundation 


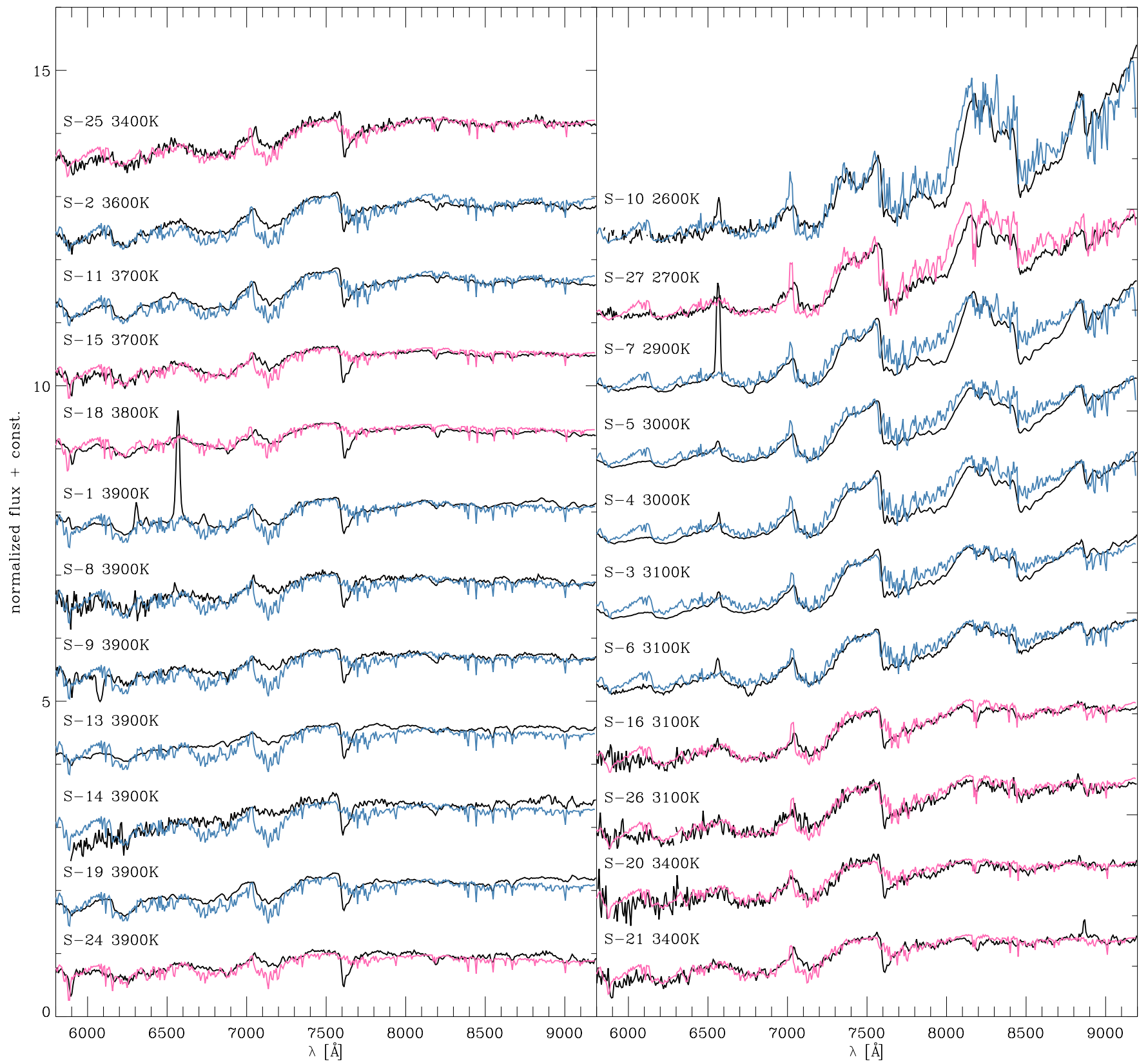

Figure 15. Spectra of the objects in Table 2 (black) and the best-fit AMES-Dusty model (green; Allard et al. 2001). The model spectra are color-coded according to the $\log (g)$ used in fitting, with blue for the low-gravity objects with $\log (g)=3.5$ or $\log (g)=4.0$ and with pink for the high-gravity objects with $\log (g)=5.0$. The spectral resolution of the models has been reduced by boxcar smoothing in order to match the resolution of our spectra. Each spectrum has been corrected for extinction but not for the atmospheric absorption. Note that the AMES-Dusty model does not extend above $T_{\text {eff }}=4000 \mathrm{~K}$; therefore, the hottest objects in our sample are not shown in this plot.

(A color version of this figure is available in the online journal.)

Ireland. B.L.M. was supported by the Spanish Plan Nacional de Astronomía y Astrofísica through project AYA 2011-30147C03-03. Support for this work also came from grants to R.J. from the Natural Sciences and Engineering Research Council of Canada.

\section{APPENDIX}

\section{MODEL FITTING PLOTS}

Figures 14 and 15 show the best-fit models from the BT-Settl and AMES-Dusty series to the spectra of the objects from Table 2. The results are summarized in Table 3. For more details please refer to Section 4.4.

\section{REFERENCES}

Alcalá, J. M., Natta, A., Manara, C. F., et al. 2014, A\&A, 561, A2

Allard, F., Hauschildt, P. H., Alexander, D. R., Tamanai, A., \& Schweitzer, A. 2001, ApJ, 556, 357

Allard, F., Homeier, D., \& Freytag, B. 2011, in ASP Conf. Ser. 448, 16th Cambridge Workshop on Cool Stars, Stellar Systems, and the Sun, ed. C. Johns-Krull, M. K. Browning, \& A. A. West (San Francisco, CA: ASP), 91

Allen, L. E., Calvet, N., D'Alessio, P., et al. 2004, ApJS, 154, 363

Allen, P. R., Luhman, K. L., Myers, P. C., et al. 2007, ApJ, 655, 1095

Allers, K. N., Kessler-Silacci, J. E., Cieza, L. A., \& Jaffe, D. T. 2006, ApJ, 644,364

Baade, D., Meisenheimer, K., Iwert, O., et al. 1999, Msngr, 95, 15

Baraffe, I., Chabrier, G., Allard, F., \& Hauschildt, P. H. 1998, A\&A, 337, 403

Baraffe, I., Chabrier, G., \& Gallardo, J. 2009, ApJL, 702, L27 
Barrado y Navascués, D., \& Martín, E. L. 2003, AJ, 126, 2997

Bastian, N., Covey, K. R., \& Meyer, M. R. 2010, ARA\&A, 48, 339

Bayo, A., Barrado, D., Stauffer, J., et al. 2011, A\&A, 536, A63

Bayo, A., Rodrigo, C., Barrado Y Navascués, D., et al. 2008, A\&A, 492, 277

Bertout, C., Robichon, N., \& Arenou, F. 1999, A\&A, 352, 574

Bessell, M. S., \& Brett, J. M. 1988, PASP, 100, 1134

Bonnell, I. A., Larson, R. B., \& Zinnecker, H. 2007, in Protostars and Planets V, ed. B. Reipurth, D. Jewitt, \& K. Keil (Tucson, AZ: Univ. Arizona Press), 149

Brown, L. D., Cai, T. T., \& Dasgupta, A. 2001, StaSc, 16, 101

Cambrésy, L. 1999, A\&A, 345, 965

Cardelli, J. A., Clayton, G. C., \& Mathis, J. S. 1989, ApJ, 345, 245

Chabrier, G., Baraffe, I., Allard, F., \& Hauschildt, P. 2000, ApJ, 542, 464

Chapman, N. L., Lai, S.-P., Mundy, L. G., et al. 2007, ApJ, 667, 288

Comerón, F. 2008, in Handbook of Star Forming Regions, Vol. II, ed. B. Reipurth (San Francisco, CA: ASP), 295

Comerón, F. 2011, A\&A, 531, A33

Comerón, F., Fernández, M., Baraffe, I., Neuhäuser, R., \& Kaas, A. A. 2003, A\&A, 406, 1001

Comerón, F., Spezzi, L., \& López Martí, B. 2009, A\&A, 500, 1045

Comerón, F., Spezzi, L., López Martí, B., \& Merín, B. 2013, A\&A, 554, A86

Epchtein, N., de Batz, B., Copet, E., et al. 1994, Ap\&SS, 217, 3

Fernández, M., \& Comerón, F. 2005, A\&A, 440, 1119

Geers, V., Scholz, A., Jayawardhana, R., et al. 2011, ApJ, 726, 23

Gehrels, N. 1986, ApJ, 303, 336

Gondoin, P. 2006, A\&A, 454, 595

Hartmann, L. 1998, Accretion Processes in Star Formation (Cambridge: Cambridge Univ. Press)

Horne, K. 1986, PASP, 98, 609

Huélamo, N., Bouy, H., Pinte, C., et al. 2010, A\&A, 523, A42

Hughes, J., Hartigan, P., \& Clampitt, L. 1993, AJ, 105, 571

Hughes, J., Hartigan, P., Krautter, J., \& Kelemen, J. 1994, AJ, 108, 1071

Jeffries, R. D. 2012, in Low Mass Stars and the Transition Stars/Brown Dwarfs (EAS Publications Series), Vol. 57, ed. C. Reylé, C. Charbonnel, \& M. Schultheis (Les Ulis: UDP Sciences), 45

Kirkpatrick, J. D., Henry, T. J., \& McCarthy, D. W., Jr. 1991, ApJS, 77, 417

Kirkpatrick, J. D., Henry, T. J., \& Simons, D. A. 1995, AJ, 109, 797

Krautter, J. 1986, A\&A, 161, 195

Krautter, J. 1992, in Low Mass Star Formation in Southern Molecular Clouds, ed. B. Reipurth (Garching: ESO), 127

Krautter, J., Wichmann, R., Schmitt, J. H. M. M., et al. 1997, A\&AS, 123, 329

Lombardi, M., Lada, C. J., \& Alves, J. 2008, A\&A, 480, 785

López Martí, B., Eislöffel, J., \& Mundt, R. 2005, A\&A, 440, 139

López Martí, B., Jiménez-Esteban, F., \& Solano, E. 2011, A\&A, 529, A108

Luhman, K. L. 2004a, ApJ, 602, 816
Luhman, K. L. 2004b, ApJ, 616, 1033

Luhman, K. L. 2004c, ApJ, 617, 1216

Luhman, K. L. 2007, ApJS, 173, 104

Luhman, K. L., Briceño, C., Stauffer, J. R., et al. 2003a, ApJ, 590, 348

Luhman, K. L., Stauffer, J. R., Muench, A. A., et al. 2003b, ApJ, 593, 1093

Makarov, V. V. 2007, ApJ, 658, 480

Martín, E. L., Delfosse, X., Basri, G., et al. 1999, AJ, 118, 2466

Mentuch, E., Brandeker, A., van Kerkwijk, M. H., Jayawardhana, R., \& Hauschildt, P. H. 2008, ApJ, 689, 1127

Merín, B., Augereau, J.-C., van Dishoeck, E. F., et al. 2007, ApJ, 661, 361

Merín, B., Jørgensen, J., Spezzi, L., et al. 2008, ApJS, 177, 551

Mortier, A., Oliveira, I., \& van Dishoeck, E. F. 2011, MNRAS, 418, 1194

Mužić, K., Scholz, A., Geers, V., Fissel, L., \& Jayawardhana, R. 2011, ApJ, 732,86

Mužić, K., Scholz, A., Geers, V., Jayawardhana, R., \& Tamura, M. 2012, ApJ, 744,134

Nakajima, Y., Tamura, M., Oasa, Y., \& Nakajima, T. 2000, AJ, 119, 873

Osterbrock, D. E. 1989, Astrophysics of Gaseous Nebulae and Active Galactic Nuclei (Mill Valley, CA: University Science Books)

Pecaut, M. J., \& Mamajek, E. E. 2013, ApJS, 208, 9

Pontoppidan, K. M., Stapelfeldt, K. R., Blake, G. A., van Dishoeck, E. F., \& Dullemond, C. P. 2007, ApJL, 658, L111

Press, W. H., Teukolsky, S. A., Vetterling, W. T., \& Flannery, B. P. 2002, Numerical Recipes in C++ : the Art of Scientific Computing (Cambridge: Cambridge Univ. Press)

Riddick, F. C., Roche, P. F., \& Lucas, P. W. 2007, MNRAS, 381, 1067

Schlieder, J. E., Lépine, S., \& Simon, M. 2012, AJ, 144, 109

Schödel, R., Merritt, D., \& Eckart, A. 2009, A\&A, 502, 91

Scholz, A., Coffey, J., Brandeker, A., \& Jayawardhana, R. 2007, ApJ, 662, 1254

Scholz, A., Geers, V., Clark, P., Jayawardhana, R., \& Muzic, K. 2013, ApJ, 775,138

Scholz, A., Geers, V., Jayawardhana, R., et al. 2009, ApJ, 702, 805

Scholz, A., Jayawardhana, R., Muzic, K., et al. 2012a, ApJ, 756, 24

Scholz, A., Muzic, K., Geers, V., et al. 2012b, ApJ, 744, 6

Schwartz, R. D. 1977, ApJS, 35, 161

Skrutskie, M. F., Cutri, R. M., Stiening, R., et al. 2006, AJ, 131, 1163

Stelzer, B., Frasca, A., Alcalá, J. M., et al. 2013, A\&A, 558, A141

Strauss, M. A., Huchra, J. P., Davis, M., et al. 1992, ApJS, 83, 29

Tachihara, K., Rengel, M., Nakajima, Y., et al. 2007, ApJ, 659, 1382

The, P.-S. 1962, CoBos, 15, 0

West, A. A., Hawley, S. L., Bochanski, J. J., et al. 2008, AJ, 135, 785

White, R. J., \& Basri, G. 2003, ApJ, 582, 1109

Wichmann, R., Bastian, U., Krautter, J., Jankovics, I., \& Rucinski, S. M. 1998, MNRAS, 301, L39

Wood, K., Wolff, M. J., Bjorkman, J. E., \& Whitney, B. 2002, ApJ, 564, 887 